UMEÅ UNIVERSITY

November 4, 2019

Department of Physics

Masters Thesis in Engineering Physics

\title{
Geoeffectiveness of Magnetosheath Jets
}

Linus Norenius lino0112@student.umu.se

Supervisor: Maria Hamrin

Examinor: Patrik Norqvist 


\begin{abstract}
In this report we present spacecraft and ground-based observations of magnetosheath jets impacting the magnetosphere, both as a case study and a statistical study. In the case study, jets were detected in the magnetosheath by the Magnetospheric MultiScale mission, MMS. By utilizing a data-based magnetospheric model (Tsyganenko T96 [29]), we estimated which jets were likely to impact the magnetopause and where they would do so. We examined ground based magnetometers, GMAGs, at the expected foot-point to the affected magnetic field line and compared this with the spacecraft observations. Theoretical transfer times for a jet to be detectable by GMAGs have been estimated and compared with the observed time delay, from detection to GMAG response, and they were in good agreement for all cases. The times found for this geoeffective response were found to be around 1-2 min, and the response in the GMAGs was in the form of a pulse with an amplitude of around $50 \mathrm{nT}$. We suggest that jets of a long enough time duration can be geoeffective in a way that they are detectable at ground level by GMAGs. It was also found that GMAGs fluctuate more during periods containing many detected jets.
\end{abstract}

We performed our statistical study with the intention of comparing fluctuations in GMAG observations during Interplanetary Magnetic Field, IMF, configurations which is suggested to be favorable for jet creation. The IMF observations was provided by the THEMIS (Time History of Events and Macroscale Interactions during Substorms) spacecraft. This was done by selecting periods of steady IMF with different orientations, and examining the GMAG variations. GMAGs were selected based on a region where most of our foot-points were found in our previous case study. We performed this study over a three year interval, and found that GMAGs observe about $2 \mathrm{nT}$ higher variation, according to their standard deviations, during radial IMF compared to northward IMF. During northward IMF we expect less effects from magnetopause phenomena, thus making it suitable to compare with radial IMF. Our statistical investigation support our findings that magnetosheath jets can be geoeffective in a way that GMAGs can detect them. 


\section{Acknowledgements}

I would like to show my appreciations to a couple of people who have helped me in various ways during my University studies and this final project. Firstly, I would like to thank my friends from the engineering physics program, if you can translate the English hockey term "grind" into Swedish, all persons concerned will know who I mean. All of you have made these last five years a some the most entertaining and I have experienced. I would also like to thank my girlfriend Felicia, for encouraging and supporting me greatly during both my studies, this project and my future plans. I also owe much gratitude to Oleksandr and Maria for incredibly valuable comments and suggestions for this project, both in regards of the physics and the writing. The entire space physics group at Umeå University have been very helpful and welcoming during my master project, and I very much look forward to becoming your colleague. This list is by no means complete, and there are many more I would like to add. 


\section{Contents}

1 Introduction 1

2 Space plasma physics $\quad 2$

2.1 Solar-terrestrial physics ..................... 4

2.2 Present day knowledge: Jets and their ground based response . . . . 6

3 Magnetospheric models $\quad 8$

3.1 Some coordinate systems . . . . . . . . . . . . . . 9

$\begin{array}{llr}4 & \text { Instrumentation } & \mathbf{1 1}\end{array}$

4.1 MMS spacecraft . . . . . . . . . . . . . . . . 11

4.2 OMNI data . . . . . . . . . . . . . . . . . . . . 11

4.3 THEMIS spacecraft . . . . . . . . . . . . . . . . . 12

4.4 Ground magnetometers and SuperMAG . . . . . . . . . . . . . 12

5 Case study of jets $\quad 14$

5.1 Jet selection . . . . . . . . . . . . . . . . . . . . . . 14

5.2 Magnetopause impact and geoeffective jets . . . . . . . . . . . 15

5.3 Observations . . . . . . . . . . . . . . . . . 17

5.4 Summary and discussion: case study . . . . . . . . . . . . 28

6 Statistical study of IMF orientation $\quad 29$

6.1 Method ......................... 30

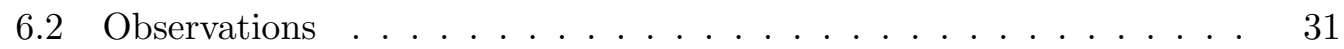

6.3 Summary and discussion: statistical study . . . . . . . . . . . 33

$\begin{array}{lll}7 & \text { Summary and conclusions } & 33\end{array}$

8 Acknowledgments $\quad 34$ 


\section{Introduction}

The universe have captivated human curiosity for a long time and still does. New planets in other solar systems, so called exoplanets, are found quite regularly. This might lead you to believe that all we know about space have reached quite far outside our own solar system. While this is true to some extent, there are still many things we do not yet know in our own solar system. In fact, the immediate neighborhood of our own planet contain a multitude of poorly understood phenomena. How can the sun's magnetic field have impact on the Earth? What connections are there between the solar wind plasma ${ }^{1}$ and space weather? Could there be space phenomena affecting us at ground level, which we have not considered yet?

The solar wind is in the very simplest description often quite stable, a steady laminar flow of plasma with slowly varying velocity. But this is not entirely true. For example, the velocity can vary greatly and include sharp changes in the interplanetary magnetic field, IMF, so called discontinuities. As this variable solar wind meets the bow shock ${ }^{2}$ high speed flows can be created. Some of these high speed flows can be related to something called jets which typically have larger velocity and density than the surrounding plasma. These jets are an example of a physical phenomena in our near vicinity that we do not fully understand. Could they cause perturbations on Earth's magnetic field, even so far down as to ground level?

In this project we have examined if these jets can cause geoeffective disturbances detectable with ground based measurements. This has been done both with a case study and a statistical investigation. The events in the case study are made up of selected events of satellite measurements from the magnetosphere and ground based magnetometers, GMAGs. Our statistical investigation compares the orientation of the IMF and the fluctuations observed by GMAGs. It is suggested that during certain IMF configurations jets are more common [23], and if we can observe more variations in the GMAG observations during such configurations, this can be explained by jets. The project is meant to act as a pre-study for further investigations on a $\mathrm{PhD}$ level. We propose that the geomagnetic fields indeed can get perturbed by some jets, and the perturbations can be observed by GMAGs in the magnetic fields.

In this report we will give a brief introduction to the subject of plasma physics, an essential part of space physics. Then we introduce solar-terrestrial physics and describe our space environment. Descriptions of space-borne and ground based instrumentation utilized for this project is presented, along with the method used for

\footnotetext{
${ }^{1} \mathrm{~A}$ gas of charged particles originating from the sun.

${ }^{2}$ The bow shock is the shock wave created as the supersonic solar wind is decelerated to subsonic speeds by Earth's magnetosphere (the magnetic bubble surrounding Earth).
} 
detection of jets and how they are correlated to ground based measurements. Then we present our results and provide some discussion. We will not discuss the creation mechanism behind jets in this project, nor their internal structure and properties, but solely focus on their geoeffectiveness.

\section{Space plasma physics}

A plasma is a gas of charged (and possibly neutral) particles and is sometimes referred to as the fourth state of matter along with solids, liquids and gasses. Increasing the energy of a solid creates a liquid, increasing the energy further produces a gas. And by raising it even more, the particles in the gas get ionized and it is this mix of ions and free electrons that is referred to as a plasma. In our everyday lives a plasma is a quite exotic state of matter, found in lightning or very high temperature flames naturally. Of course it can be generated in laboratories now a days but otherwise it is relatively rare. In our visible universe however, almost everything is in the plasma state. The solar wind plasma for example, is composed mainly of hydrogen and helium ions and electrons. A plasma does not need to be completely ionized, as in the solar wind, to be referred to as a plasma. Our ionosphere for example, is only partly ionized, but still qualifies as a plasma. The fact that a plasma is made up of charged particles implies that it interacts highly with the electromagnetic fields. Hence, forces from collisions or gravity are often neglected when discussing ideal plasmas and electromagnetic forces are generally dominant for the dynamics of a plasma.

There are three important numbers or parameters when talking about plasmas: the Debye length $\lambda_{D}$, the plasma parameter $\Lambda$ and plasma electron frequency $\omega_{p}$. The debye length is the distance at which the fields generated by a charged particle become negligible due to shielding from the surrounding particles. The plasma parameter is defined as the number of particles within a sphere of Debye length radius. If the equilibrium of the plasma is disturbed by some external force, the electrons, which are much more mobile than the heavier ions, will start to accelerate to attempt to restore charge neutrality. The frequency at which this occurs is the plasma electron frequency. To qualify as an ideal plasma the following relations must hold

$$
\begin{aligned}
& \lambda_{D}<<L \\
& \Lambda>>1 \\
& \omega_{p} \tau>1,
\end{aligned}
$$

where $L$ is the typical length scale of the system in consideration and $\tau$ is the mean time between collisions with neutrals. That is, a plasma which truly is governed by large scale collective forces and collisions are of little importance. The above limits ensures that Debye shielding can occur, that the plasma is quasi-neutral and that 
collisions are infrequent enough to be neglected.

To describe the dynamics of plasmas, there are three common approaches, the single particle, statistical and fluid approach.

By considering each single particle alone, useful insight of the behavior of a plasma can be obtained. However, this can be an impractical approach since there is no trivial way to describe macroscopic variables, such as charge density and temperature, in terms of single particle trajectories. The particle density within the solar wind plasma is about $10^{7} \mathrm{~m}^{-3}$ and the relevant length scales are huge (in the order of Earth's radius $R_{E} \approx 6300 \mathrm{~km}$ ), and keeping track of all those particles quickly becomes rather difficult.

The statistical approach, often called the kinetic approach, models the plasma using a 6-dimensional distribution function which is a function of three spatial and three velocity variables. The distribution function is governed by the so called Vlasov equation [30] (if collisions can be ignored). From the distribution function different physical quantities such as the bulk velocity and particle density can be derived. Neither of these two methods have been used for this project, but instead the fluid approach have been used.

Instead of bothering about the evolution of the distribution function, the fluid model tracks the macroscopic quantities which can be derived from it. These quantities such as the velocity, density and temperature, can be related to fluid theories. Magnetohydrodynamics (MHD) was a term first proposed by Hannes Alfvén in 1942 [1], which can be derived from a more general theory called multi fluid theory. In the multi fluid theory each particle species is seen as a separate fluid whereas in the MHD theory the plasma is treated as a single fluid containing both electrons and ions.

From the MHD equations (which includes both the Navier-Stoke's fluid equations and Maxwell's equations) we can derive a typical speed for information to propagate through a magnetized plasma, the Alfvén velocity (see, for e.g. [4]). Several wave modes corresponding to different velocities in a plasma exists, but for our case the shear Alfvén wave will be considered. After some simplifying assumptions ${ }^{3}$, the Alfvén velocity can be calculated as

$$
v_{A}=\frac{B}{\sqrt{\mu_{0} n_{i} m_{i}}},
$$

\footnotetext{
${ }^{3}$ Assuming a cold plasma and neglecting the electron mass.
} 
where $B$ is the magnetic field strength, $\mu_{0}$ the permeability of free space, $n_{i}$ the ion number density and $m_{i}$ the mass of the ion species in consideration.

\subsection{Solar-terrestrial physics}

Solar-terrestrial physics is an important application of plasma physics and a large part of space physics. It concerns the coupling between the solar wind and magnetic fields from the sun to the Earth and its fields, how they can interact and affect each other. Effects from the solar wind on Earth's magnetic field is a major part of this. The solar wind is a stream of plasma originating from the heated upper atmosphere of the sun and propagate throughout the solar system with supersonic speeds, about 300-500 km/s and with a density of about $10 \mathrm{~cm}^{-3}$ at the Earth's orbit. It consists mainly of ionized hydrogen, helium and electrons. When the solar wind is decelerated to subsonic speeds by obstacles of some kind, such as the Earth's magnetosphere, a shock is created, the bow shock (see Fig. 1).

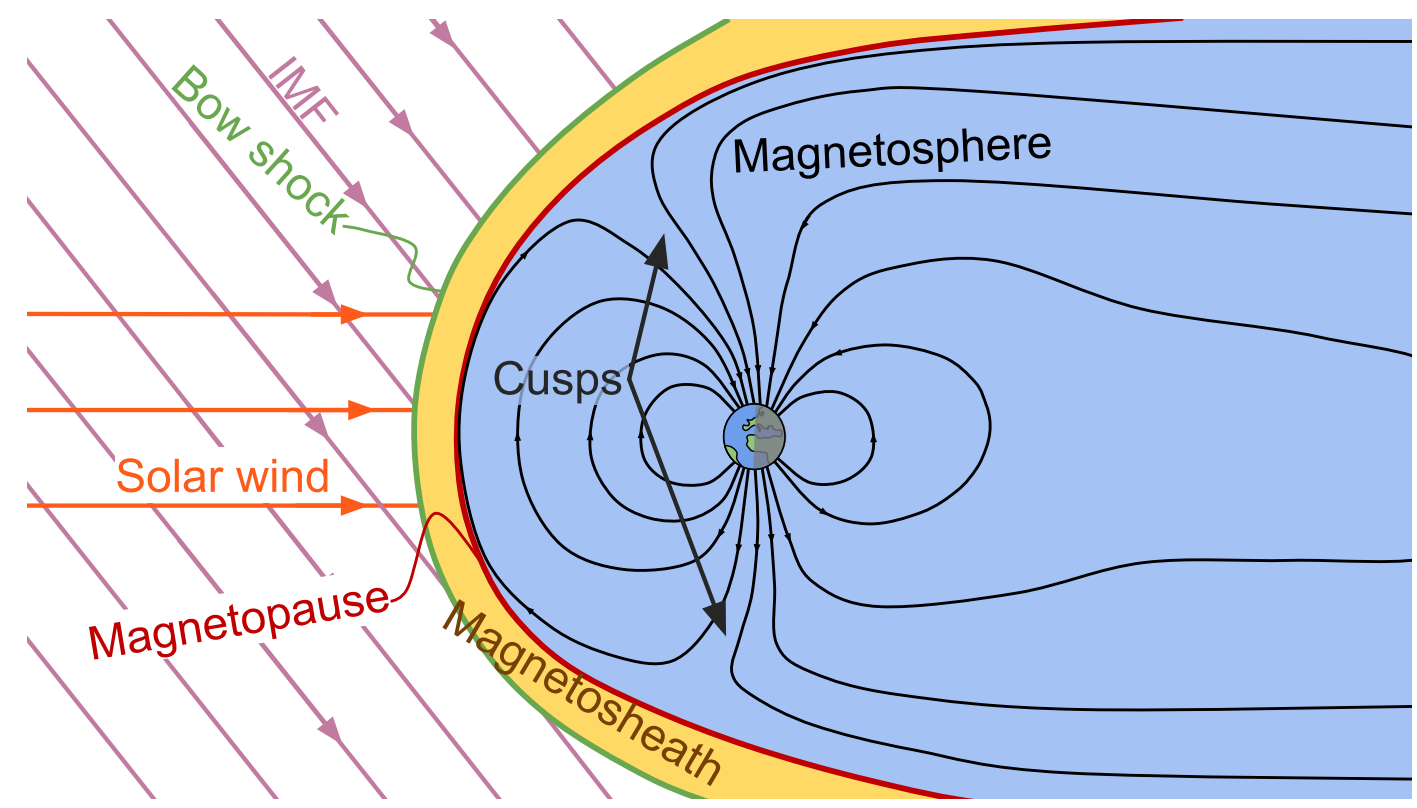

Figure 1: Earths magnetosphere (light blue) and the magnetic field lines (black) surrounded by the magnetopause (red) and the magnetosheath (yellow). The solar wind (orange) is decelerated at the bow shock (green) with the IMF outside (magenta). The cusps is indicated with two black arrows. The image is not to scale.

The magnetosheath, a turbulent region with shocked plasma and higher temperature, lies between the bow shock and the magnetopause. The magnetosheath contains 
higher density plasma but lower magnetic field strength compared to the magnetosphere.

The final boundary before the magnetosphere is the magnetopause, behind which the regular geomagnetic field exists as we mostly know it. In the magnetosphere the magnetic field strength is much higher compared to in the magnetosheath and in the solar wind but much lower plasma velocities. On the nightside, the magnetosphere stretches far out into interplanetary space and is referred to as the magnetotail due to its shape.

In this project we will focus on magnetosheath jets. It is suggested that they can be created at the bow shock and propagate towards the Earth and possibly collide with the magnetopause causing geoeffective disturbances and introduce solar wind plasma into the magnetosphere. Other ways for external plasma to enter the magnetosphere is through a process called magnetic reconnection [4, 28], Figure 2 shows a sketch of this.

1)

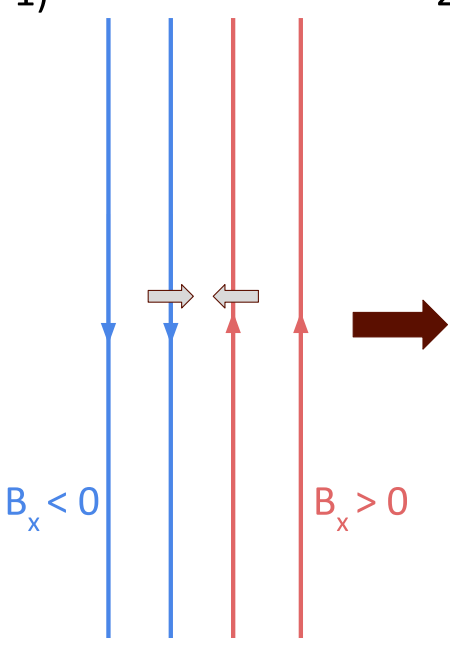

2)

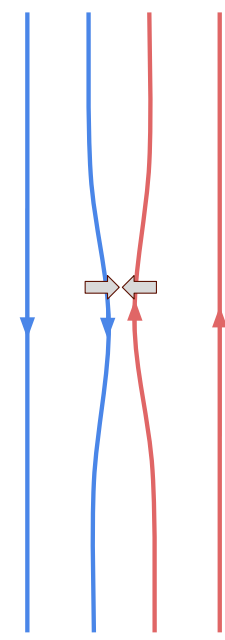

3)

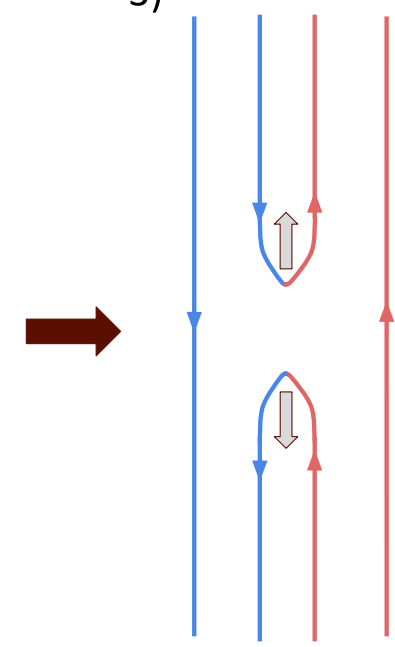

Figure 2: Magnetic reconnection happens when field lines with opposite direction approach each other, eventually merging and rearranging the field topology. Large amounts of field energy is then converted to particle kinetic energy.

Magnetic reconnection is a phenomena where magnetic field energy hastily converts to kinetic energy in the plasma. The dayside magnetopause is a region where re- 
connection commonly can occur, as well as in the magnetotail. When reconnection happens at the dayside magnetopause, energetic plasma can enter the magnetosphere introducing large amounts of momentum and energy to the dayside ionosphere (the ionized part of Earth's atmosphere). This is more common during southward IMF (see Fig. 3a). However, reconnection can occur on the dayside during other IMF orientations as well (Fig. 3b-d). During northward IMF for example, reconnection occurs at the polar cusps. Radial ${ }^{4}$ IMF gives rise to reconnection at the day side magnetopause and at one of two the cusps, which one depends on whether the field is radially inward or outward.
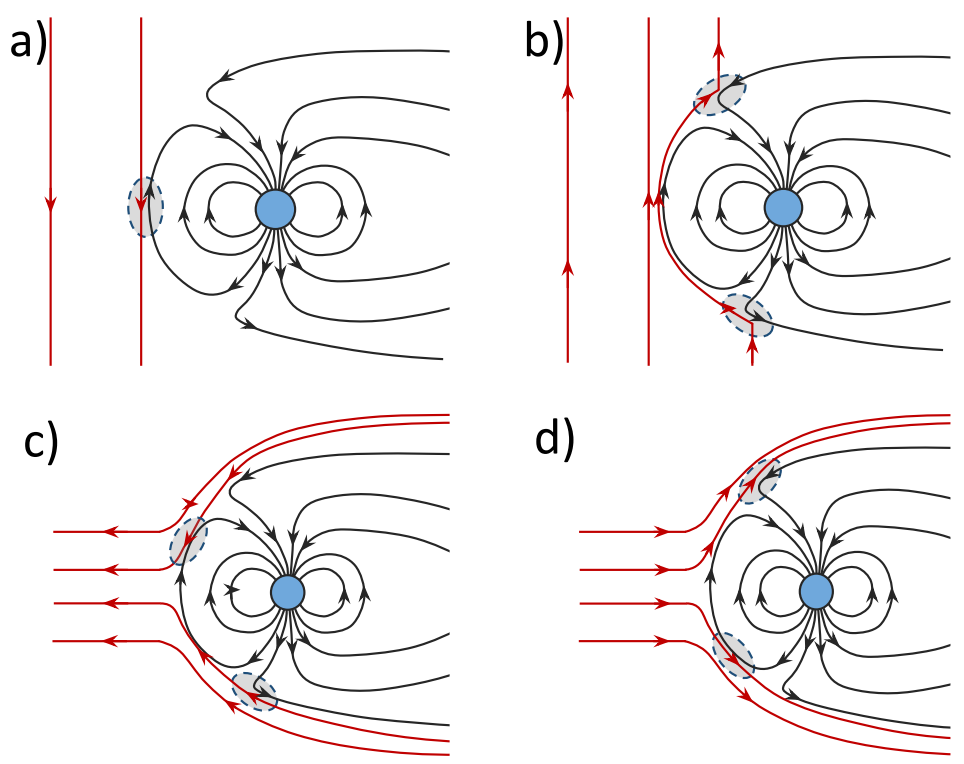

Figure 3: Day side reconnection occurs at different sites depending on the IMF orientation, a) southward, b) northward, c) radially inward (sunward), d) radially outward (Earthward). Note that the image is for illustrative purposes and not to scale.

\subsection{Present day knowledge: Jets and their ground based response}

Another way for plasma to enter the magnetosphere is through impulsive penetration [12], where some magnetosheath transient with higher velocity than the background bulk velocity can penetrate through the magnetopause and enter the magnetosphere. In the literature similar transients affecting the magnetopause have been reported, for example, Dmitriev et al. $(2012,2015)[9,8]$ reported motion of

\footnotetext{
${ }^{4}$ Radial IMF means that the angle between the field lines and the sun-Earth line is less than $30^{\circ}$.
} 
the magnetopause likely caused by an impacting jet. During this event, they suggest that magnetosheath plasma managed to penetrate into the magnetosphere and the plasma remained for about $10 \mathrm{~min}$. Such transients have been denominated magnetosheath jets, high speed jets, plasmoids or density enhancements but Karlsson et al. (2015) [18] suggest that they could be a subset of similar things. They are often characterized by higher dynamic pressure than the surrounding plasma, where the dynamic pressure is given by $P_{d y n}=\rho v^{2}, \rho$ being the mass density and $v$ the velocity.

In this project we will investigate what effect these jets could have on ground level, or their geoeffectiveness. Previous studies report ground-based observations which might be related to jets. Hietala et al. [15] for example, used spacecraft observations and reported multiple events with inward motion of the magnetopause followed by jets, suggesting that the jets might be impacting the magnetopause causing perturbations and waves on the magnetopause. Additionally, they compared this with simultaneous ground based observations, which recorded localised and short-lived pulses. The location and timing of these pulses suggest that they were caused by jets. Archer et al. [3] also reported magnetopause motion caused by a jet (pressure pulse in the article). GMAG and ground based radar observations indicated perturbations induced by this jet impacting the magnetopause. Furthermore, they suggested that the magnetopause acts as a low-pass filter, meaning that only jets of long enough time duration might cause perturbations detectable in ground based observations. Han et al. [14] focuses on something called day-side throat aurora ${ }^{5}$. They suggest that jets might be a cause of these auroras.

The creation mechanism of these jets are still not fully understood and a multitude of explanations have been proposed. It is generally agreed that they are more commonly created at what is called a quasi-parallel bow shock. That is, when the angle between the bow shock normal and the IMF is $<45^{\circ}$. This occurs around the subsolar point ${ }^{6}$ during radial IMF. Hence, jets created during this IMF configuration are more likely to impact the day-side magnetopause. At a quasi-parallel bow shock, a lot of different phenomena occur which might be related to the creation of jets. For example, Plaschke et al. [22] suggest that ripples on the bow shock, which are common at a quasi-parallel bow shock, might deflect incoming solar wind plasma in a way which generates a jet in the magnetosheath. Discontinuities in the solar wind, interacting with the bow shock have also been suggested to create jets [16].

\footnotetext{
${ }^{5}$ Auroras are mostly generated and visible on the nightside. Dayside auroras might be related to magnetopause mechanisms. Throat auroras are called that due to their particular shape on the sky.

${ }^{6}$ The point on a planet, or bow shock in this case, where the sun appears directly above an observer.
} 


\section{Magnetospheric models}

Earth's magnetic field depends on both the inner magnetic fields, generated inside the Earth, and on fields generated by different magnetospheric current systems, see Figure 4. A model of this system should reasonably well be able to replicate the combined fields associated with magnetopause compression and increases/decreases in the currents. The Tsyganenko T96 model is a data-based model of the Earth's magnetic fields, based on mathematical modeling and data fitting from spacecraft and GMAGs [29]. It works much like a polynomial fit for a function, where measured parameters acts as weights for different parts of the function. It treats the external geomagnetic fields, $\mathbf{B}_{E}$, as a sum of all the fields generated by the major magnetospheric current systems

$$
\mathbf{B}_{E}=\mathbf{B}_{M P}+\mathbf{B}_{R C}+\mathbf{B}_{T}+\mathbf{B}_{F A C}+\mathbf{B}_{I n t},
$$

where the terms on the right hand side represents contributions from the magnetopause current system (MP), ring current (RC), tail current (T), field aligned currents (FAC) and a term due to partial penetration from the IMF into the geomagnetic fields (Int). The T96 model can predict the geometry of the magnetosphere depending on different upstream solar wind conditions.

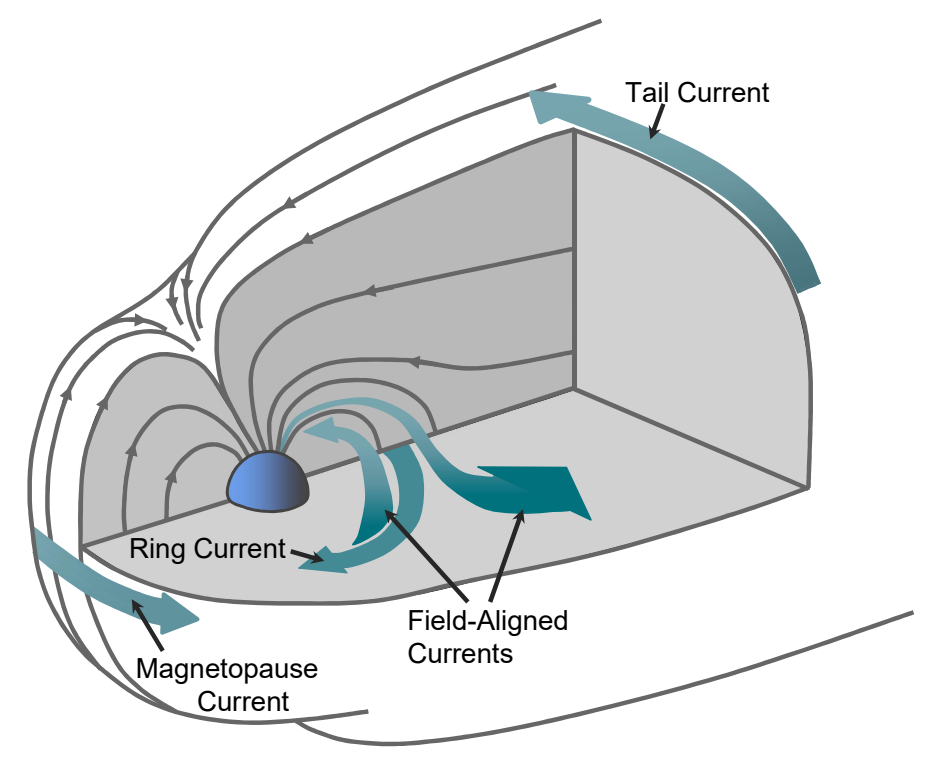

Figure 4: Overview of different current systems in the magnetosphere (not to scale), adapted from [4]. 
For our case study, a prepared routine for implementing the T96 model have been used. It takes as inputs averaged values of the dynamic pressure of the solar wind, $B_{y}$ and $B_{z}$ from the IMF, the velocity of the solar wind and something called the Disturbance storm index, Dst ${ }^{7}$. These inputs should be averaged over the preceding hour. However, as a first approximation we have used instant values at the nominal bow shock. This can affect the results from the model, and for future studies this should be addressed.

\subsection{Some coordinate systems}

A common coordinate system for space physics is the Geocentric Solar Ecliptic system, or GSE (see Figure 5). This is a co-rotating system following the Earths orbit around the sun along the year. If we draw a straight line between the sun and the Earth we have our $x$-axis. The $z$-axis is positive in the north direction and perpendicular to the ecliptic plane, that is, the plane wherein Earths orbit lies. Then the $y$-axis closes the set to form a right-handed system, pointing towards dusk, (remember that the Earth is rotating anti-clockwise around the $z$-axis).

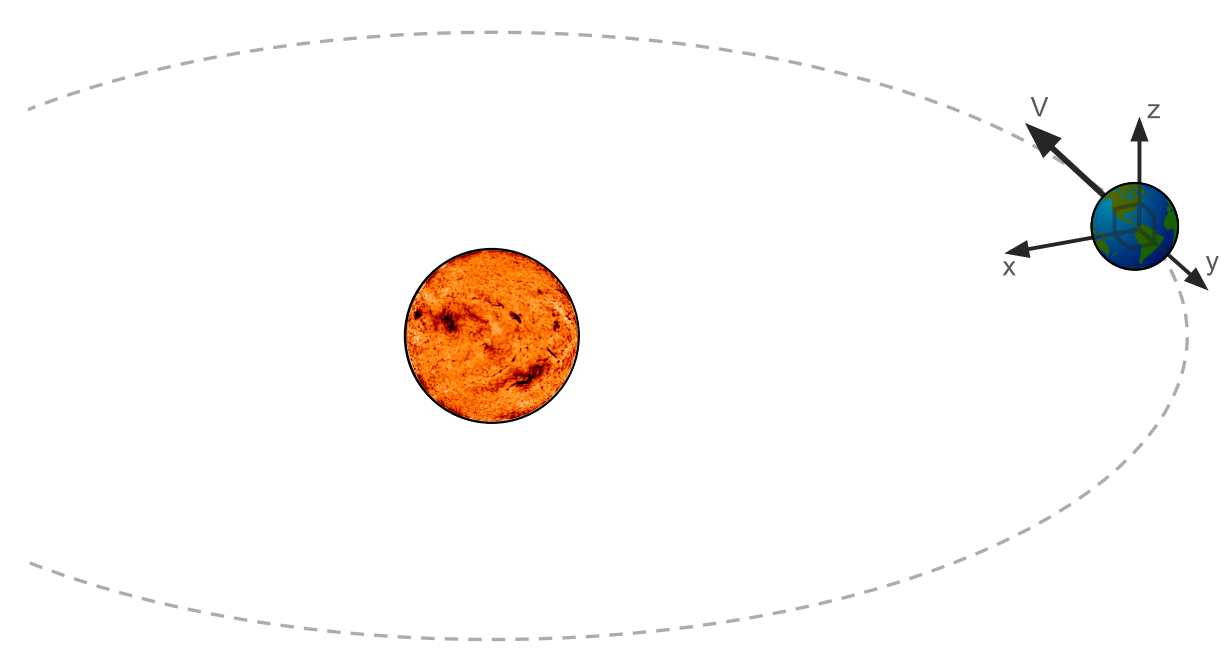

Figure 5: Sketch of the GSE coordinate system (not to scale).

\footnotetext{
${ }^{7}$ Dst index is derived from 4 GMAG stations close to the equator. These stations monitor the ring current (see Fig. 4) which gives an indication geomagnetic activity.
} 
The coordinate system used for GMAGs in this report is called Geomagnetic coordinates. This system is fixed with respect to the geomagnetic fields and very similar to Geographic coordinates with latitude, longitude and height above the ground (see Fig. 6). The basis vectors are called $N, E$ and $Z$ here but sometimes $x, y$ and $z$ is used as well. The $N$ vector points directly towards the magnetic north and the $E$ vector to the magnetic east. The $Z$ vector then points vertically down to form a right handed system.

Too keep track of time in a consistent manner in space physics, one often use Coordinated Universal Time, or UTC. This is very similar to the Greenwich Mean time, GMT, which is defined as the mean solar time in Greenwich London. GMT is based on the Earth's rotation and the suns apparent location on the sky, this does however introduce some errors over time. UTC accounts for this discrepancy by introducing leap-seconds once every 19 months. Spacecraft and GMAG data presented in this report will all use UTC.

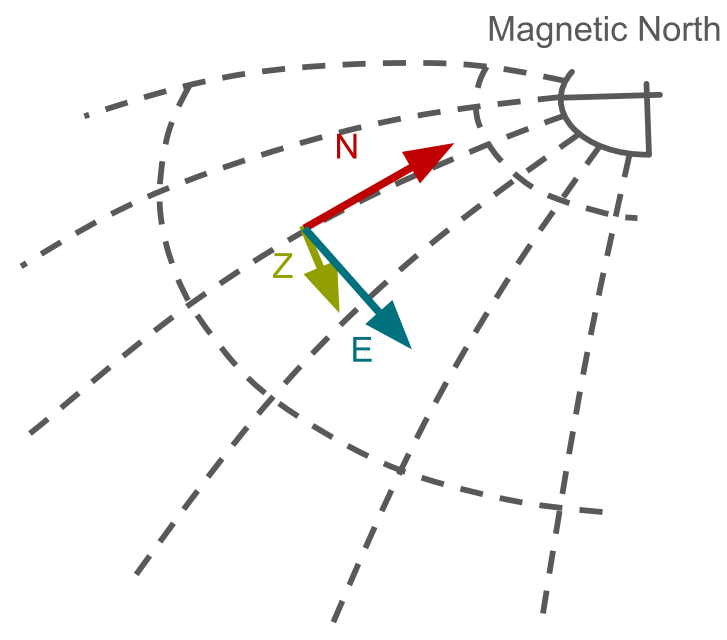

Figure 6: Image depicting the Geomagnetic coordinates on the surface of a sphere. 


\section{Instrumentation}

\subsection{MMS spacecraft}

The Magnetospheric MultiScale mission, MMS, was launched by NASA on 13 March 2015 from Cape Canaveral [6]. The mission consists of 4 identical spacecraft, orbiting clockwise in the equatorial plane, with the scientific purpose of investigating regions with magnetic reconnection: at the dayside magnetopause and in the magnetotail. During its first 1.5 years of primary mission time, MMS was flying in an elliptical orbit with apogee $^{8}$ of about $13 R_{E}\left(1 R_{E} \approx 6371 \mathrm{~km}\right.$ is the mean radius of the Earth). This allowed MMS to efficiently monitor common reconnection sites, at the day side magnetopause and in the magnetotail.

MMS carries 11 scientific experiments, capable of measuring for example particle velocities, densities and electromagnetic fields. Although designed to monitor reconnection, MMS provides important observations related to other phenomena than reconnection. For this projects case study, we have mainly used data from the flux gate magnetometer, FGM [27], and the fast plasma investigation, FPI [24]. FPI in turn consists of 2 different sensors, the dual ion spectrometer, DIS, and the dual electron spectrometer, DES, to measure ions and electrons separately.

\subsection{OMNI data}

NASA provides solar wind measurements from multiple spacecraft, especially Wind [20] and the Advanced Composition Explorer [7], ACE, both orbiting at the L1 Lagrangian point between the sun and the Earth at a distance of $\sim 200 R_{E}$ from the Earth. The data can be extracted from NASA/GSFC's OMNI data set through OMNIWeb [19] and contains solar wind velocities and IMF observations to name a few. More spacecrafts contribute to this data set whenever available.

NASA provides the OMNI data time-shifted, or propagated, from L1 to nominal the bow shock by considering the local solar wind velocity and the distance to the nominal bow shock. However, since the distance between the individual spacecraft and between L1 and the nominal bow shock is very large $\left(\sim 100 R_{E}\right.$ between ACE and Wind) it can be difficult to obtain accurate solar wind properties at the bow shock. The individual spacecraft can sometimes disagree, leading to ambiguities on what observations to propagate. Furthermore, the solar wind velocity varies which complicates the propagation from L1. For an event study, the solar wind properties can be confirmed by additional spacecraft. But for a more prolonged statistical

\footnotetext{
${ }^{8}$ Apogee is the point in an elliptical orbit furthest away from the planet, perigee is the closest point.
} 
investigation, one have to take these issues in consideration. For this reason we used observations from THEMIS, Time History of Events and Macroscale Interactions during Substorms, space craft for our statistical investigation. Since they can provide similar observations much closer to the bow shock than those from OMNI.

\subsection{THEMIS spacecraft}

THEMIS is composed by five identical spacecraft which was launched by NASA on 17 February 2007 in equatorial orbits [5]. Similar to previous mentioned spacecraft, THEMIS is also capable of measuring both particle velocities, densities and magnetic fields. As of 2011, the two spacecraft with the highest apogee, THEMIS B and C, were transferred to lunar orbits instead, while the remaining three still orbits the Earth.

THEMIS B and C, now called ARTEMIS, Acceleration Reconnection Turbulence and Electrodynamics of Moon's Interaction with the Sun, can also provide valuable upstream solar wind conditions. The moon orbits the Earth at a distance of $\sim 60 R_{E}$, much closer than ACE and wind at L1. This reduces the issue involved with propagating solar wind observations to the bow shock, and in turn increases the likelihood of obtaining accurate solar wind properties. We performed a very simple propagation of the THEMIS observations to the nominal bow shock, in our statistical study. This was done by time-shifting the observations by an amount $t=\left\langle x_{\text {THEMIS }}\right\rangle /\left\langle v_{S W}\right\rangle$, $x_{\text {THEMIS }}$ being the average distance between THEMIS and the nominal bow shock along the $x_{G S E}$ axis and $\left\langle v_{S W}\right\rangle$ the average solar wind velocity.

\subsection{Ground magnetometers and SuperMAG}

SuperMAG is a world spanning collaboration of both scientific organizations and various national agencies [10]. They gather data from over 300 GMAGs (see Fig. 7) for online plotting and research purposes [26]. The data they provide are rotated into a common coordinate system and cleaned from possible errors. In addition, they also proved the data with subtracted baselines, yearly and daily [11]. 


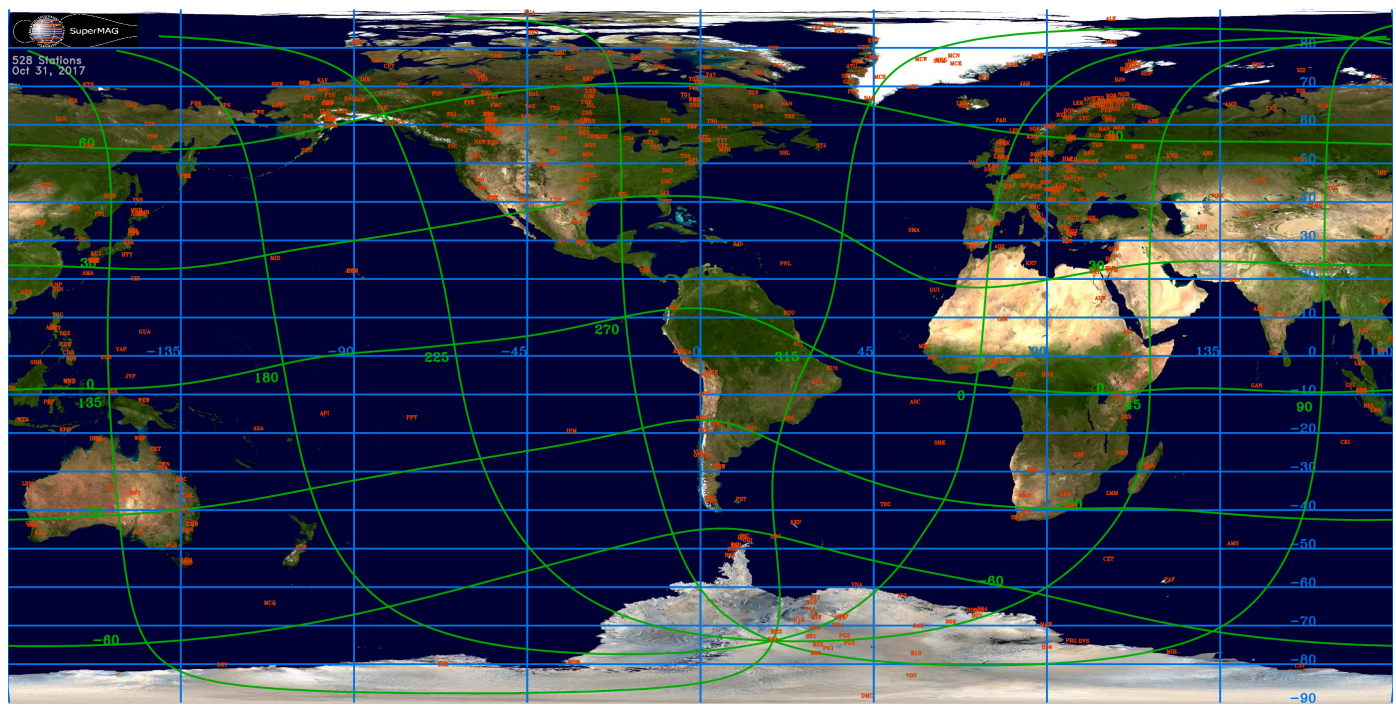

Figure 7: World map projection showing ground based magnetometers (red) from the SuperMAG collaboration [26]. Green and blue lines indicate geographic and geomagnetic coordinates respectively.

Each station is given a three letter code by the International Association of Geomagnetism and Aeronomy, IAGA. Our statistical study included GMAGs from all over the northern hemisphere and for our case study certain GMAG appeared more often. All stations used in our case study are presented in Table 1 with their IAGA codes, names and geographical coordinates.

Table 1: GMAG stations involved in the case study with their names and geographical coordinates.

\begin{tabular}{|l|l|c|c|}
\hline $\begin{array}{l}\text { IAGA } \\
\text { code }\end{array}$ & Station name & $\begin{array}{l}\text { Geographic } \\
\text { latitude }\end{array}$ & $\begin{array}{l}\text { Geographic } \\
\text { Longitude }\end{array}$ \\
\hline DED & Deadhorse & 64.05 & 211.21 \\
\hline NAL & New Aalesund & 78.92 & 11.95 \\
\hline LYR & Longyearbyen & 78.2 & 15.83 \\
\hline HOP & Hopen Island & 76.51 & 25.01 \\
\hline BJN & Bear Island & 74.5 & 19.2 \\
\hline HRN & Hornsund & 77 & 15.6 \\
\hline BRW & Barrow & 71.3 & 203.25 \\
\hline VIZ & Vieze Island & 79.48 & 76.98 \\
\hline BBG & Barentsburg & 78.04 & 14.23 \\
\hline DMH & Danmarkshavn & 76.77 & 341.37 \\
\hline
\end{tabular}




\section{Case study of jets}

In this section we will introduce our case study of geoeffective jets. First we will present our jet selection criteria, which is a combination of three separate criteria, based on MMS and OMNI observations. Then we discuss how to estimate which jets seem likely to impact the magnetopause, and where on Earth at ground level one might expect detectable perturbations in GMAGs. We also present theoretical estimates of the time delay, given that MMS have located a jet, to when it can be expected to be seen in GMAG observations.

\subsection{Jet selection}

Due to the many different suggested creation mechanisms for jets, there is also many different ways of defining a jet from spacecraft data. Figure 8 shows all three criteria we have used. The first panel shows a ion energy-time spectrogram from MMS, it displays the amount of energy flux observed.

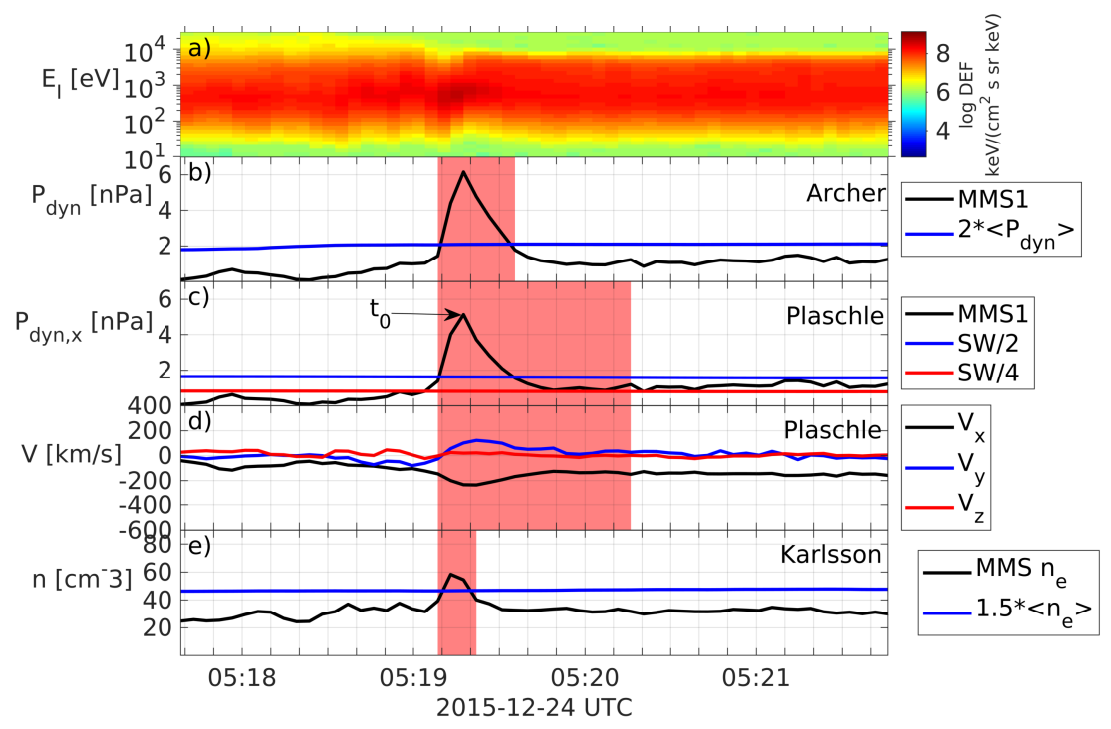

Figure 8: Example of jet selection. a) Ion energy-time spectrogram from MMS, b) Dynamic pressure (black) and background dynamic pressure obtained as a 20 min running average (blue), c) magnetosheath dynamic pressure in the $x_{G S E}$ direction (black) and from OMNI data divided by 2 and 4 (blue and red), d) ion velocity in $x_{G S E}, y_{G S E}$ and $z_{G S E}$ (black, red and blue), e) electron density (black) and background density obtained as a $500 \mathrm{~s}$ running average (blue). Jets according to the different criteria, mentioned in the text, are highlighted. 
The $y$-axis shows the energy while the $x$-axis shows the time of the spacecraft clock. The latter one also corresponds to spatial position through the relative motion between the spacecraft and the plasma. The color-bar indicates the amount of observed flux. So around 05:20 UTC, we see a slightly darker red in the 800-1000 keV region, as well as a more bright green color at the lower energies. This is a indication that more of the particles have similar energies, or velocities, at the time, which is typical for a magnetosheath jet. The following panels show time series of observed quantities from MMS and OMNI; dynamic pressure (total in b and due to $v_{x}$ in $\mathrm{c}$ ), velocity and electron density respectively.

One of our used criteria comes from Archer et al. (2013) [2] locate jets by considering the dynamic pressure, $P_{d}$, in the magnetosheath. In a jet, the dynamic pressure must exceed twice the value of the dynamic pressure in the background magnetosheath (see Fig. 8b). Where the background value is calculated by a 20 min running average.

Plaschke et al. (2012) [21] on the other hand, only look at the dynamic pressure due to the velocity in the $x_{G S E}$-direction. This needs to be higher than half the dynamic pressure (in the $x_{G S E}$-direction) in the solar wind, as obtained from OMNI $P_{d, x}>P_{d, x}^{S W} / 2$ (see Fig. 8c). If we define $t_{0}$ as the time of the maximum magnetosheath pressure, the duration of the jet is defined as the times when $P_{d, x}>P_{d, x}^{S W} / 4$ before and after $t_{0}$. Further, the ion velocity in the $x_{G S E}$-direction must be negative during the entire jet (Fig. 8d). This criteria also requires that 1 min before and after the jet, as well as the actual jet, must lie within proper magnetosheath measurements.

Finally, Karlsson et al. (2012) [17] only consider the electron density $n_{e}$ to classify jets. The electron density must be higher than 1.5 times the background density in the magnetosheath (Fig. 8e). Similarly to Archer, the background value is calculated by a $500 \mathrm{~s}$ long moving average.

For our events, we require that at least two of the above three criteria must be fulfilled simultaneously to be considered a jet. In the presented events we also only include events where we see GMAG disturbances possibly related to a jet impact.

\subsection{Magnetopause impact and geoeffective jets}

To determine which jets were likely to impact the magnetopause, and where, we propagate detected jets along their plasma velocity until we get an impact with the magnetopause from the Tsyganenko T96 model (Sec. 3). We define the magnetopause from T96 as the first closed field line (when the field line ends up in the ionosphere with one end on the northern/southern hemisphere respectively) along the path of the jet. This tells us which jets that are likely to impact the magne- 
topause, where they would impact it and which field line will be affected (red line in Fig. 9).

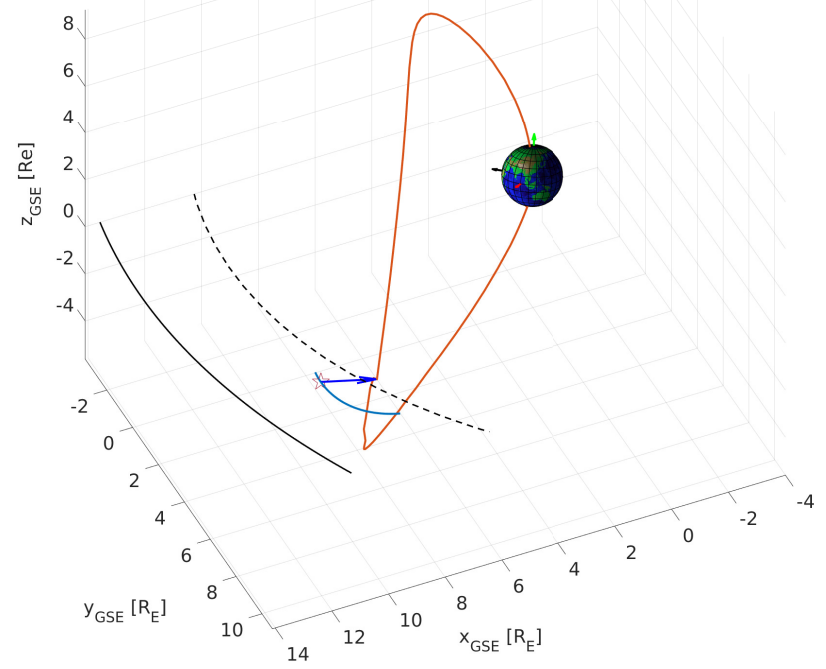

Figure 9: 3D overview of MMS (orbit in blue) detecting a magnetosheath jet (star) which propagate toward (blue arrow) and impact the nominal magnetopause from the T96 model. The affected field line (red) tracing back to Earth is seen. The black (dashed) line depicts a model bow shock (magnetopause) from [25].

To examine the geoeffectiveness of jets we select observations from GMAGs in close vicinity to where the affected field-line ends in the ionosphere, the foot-point. Given a foot-point, GMAGs within a certain distance from it was selected and compared with MMS data. Since the spatial resolutions of GMAGs vary greatly across the Earth, see Figure 7, the actual distance from foot-point to the closest GMAG varies from case to case. Only GMAGs on the northern hemisphere were chosen for this study, due to the lack of GMAG on the southern hemisphere.

To get an indication of whether or not GMAG responses are caused by a impacting jet, we estimate the time needed for the jet to propagate to the magnetopause, and the time needed for a signal to travel down to the ionosphere. We define the time 
from jet detection to the T96 magnetopause impact as $t_{\text {Imp }}$ and the time needed for the signal to propagate along the field-line down to Earth's ionosphere as $t_{A}$, the Alfvénic transfer time. The total time from detecting a jet to an expected GMAG response is then the sum of these two, $t_{t o t}=t_{\text {Imp }}+t_{A}$. The first of these two, $t_{\text {Imp }}$, can easily be calculated as $d / v_{\text {jet }}$, where $v_{\text {jet }}$ is the speed of the jet and $d$ the distance between the point of the jet detection and the point where T96 indicate magnetopause impact. And similarly for $t_{A}=d_{F L} / v_{A}$, where $d_{F L}$ is the length of the field line and $v_{A}$ the Alfvén velocity (see Sec. 2 eq. 1).

To calculate $v_{A}$ we use values for the magnetic field strength $B$ from T96 and a constant value for the density $n=2 \cdot 10^{5} \mathrm{~m}^{-3}$. To get a more accurate estimate for $n$ along the entire field line one cannot assume that it remains constant. But if one exclude the ionosphere from the path and stops well before it, $n$ can be considered constant [13]. For this reason we instead estimate the time needed for a signal to reach $2 R_{E}$ altitude above ground. The remaining $2 R_{E}$ is assumed not to effect the total time with any significance. In general we obtained $t_{A} \approx 40 \mathrm{~s}$ for most of our selected cases.

In our case study, this theoretical transfer-time is compared with the observed time. The actual observed time delay from the jet detection to the GMAG response is measured from the center of the jet to the beginning of the slope of the GMAG response or pulse. The center of the jet had to be estimated by eye from the plots, since the different criteria may well disagree of when there is a jet, as is clear from Figure 8.

\subsection{Observations}

Below, three selected events will be displayed and discussed regarding GMAG response to jets. More similar events as those shown here have been found but will not be presented here. These events will be subjects for later studies instead.

The first event is from 2015-10-22 UTC, where MMS was moving in an outbound orbit and crossed the magnetopause into the magnetosheath at around 06:00 UTC. This can be seen in Figure 10a, where there is a sudden change in the energy flux. There seems to be at least one magnetopause crossing slightly before the final crossing, indicated by a thin stripe in the spectrogram, implying that the magnetopause is moving relative to the spacecraft. The magnetopause crossing is also visible in panels d-f, showing a large increase in plasma density and velocity, as well as a large decrease in the magnetic field strength as the spacecraft moves from the magnetosphere into the magnetosheath. 
The OMNI observations propagated to the nominal bow shock (Fig. 10g) indicate that the IMF configuration was radial during the times around 07:00-07:50, due to that $B_{S W, x}$ was the dominating component (see Sec. 2.1 footnote 4 ). This IMF configuration results in a quasi-parallel bow shock close to the nose, according to the propagated OMNI data. However, the spectrogram (Fig. 10a) suggests that the bow shock was quasi-parallel during a longer time than suggested by the OMNI data. This can be seen by the amount of high energy flux recorded by MMS at about 06:50-08:20 UTC.

From about 07:50 UTC, $B_{S W, z}$ was increasing steadily (Fig. 10g). $B_{S W, z}$ had eventually increased enough so that the bow shock turned into a quasi-perpendicular one at $\sim 08: 10$ UTC. At this time when $B_{z, G S E} \approx B_{x, G S E}$, we can see in panel a, that the amount of high energy particles is less compared to previously. Less high energy particles can also be observed at around 06:30-06:40 UTC when $B_{S W, z}$ changed from about $-4 \mathrm{nT}$ to $+3 \mathrm{nT}$ for a brief period. Again the amount of high-energy flux drops during this short period.

The jet of interest (highlighted red in Fig. 10) is detected in the MMS data at around the same time as the quasi-parallel bow shock changes into a more quasiperpendicular one at $~ 08: 25$ UTC. The Plaschke and Archer criteria (Fig. 10b-c) considered this as a jet while the Karlsson criteria did not (panel d). At the time of the jet, we observe a increase in $-v_{x}, v_{y}$ (Fig. 10e) and the electron density (Fig. 10d). The magnetic field as observed by MMS (Fig. 10f) suddenly changed at the jet detection, for example $B_{y}$ and $B_{z}$ can be seen to increase. This is an indication that MMS entered another plasma regime.

Panels h-i in Figure 10 show the projections in the $x z_{G S E^{-}}$and $x y_{G S E}$-planes of MMS orbit (blue line) during the time interval displayed in Figure 10a-g. The location of the jet at around 08:25 UTC is indicated by a star and its velocity is shown as a blue arrow. This jet was estimated to impact the nominal magnetopause (black dashed line in panel $\mathrm{h}-\mathrm{i}$ ) around $15 \mathrm{~s}$ after detection. The affected field line (red line in panel h-i) had a northern foot-point at $79.7^{\circ}$ geographical latitude and $97.3^{\circ}$ longitude where the closest available GMAG at the time was VIZ (see Table 1). 

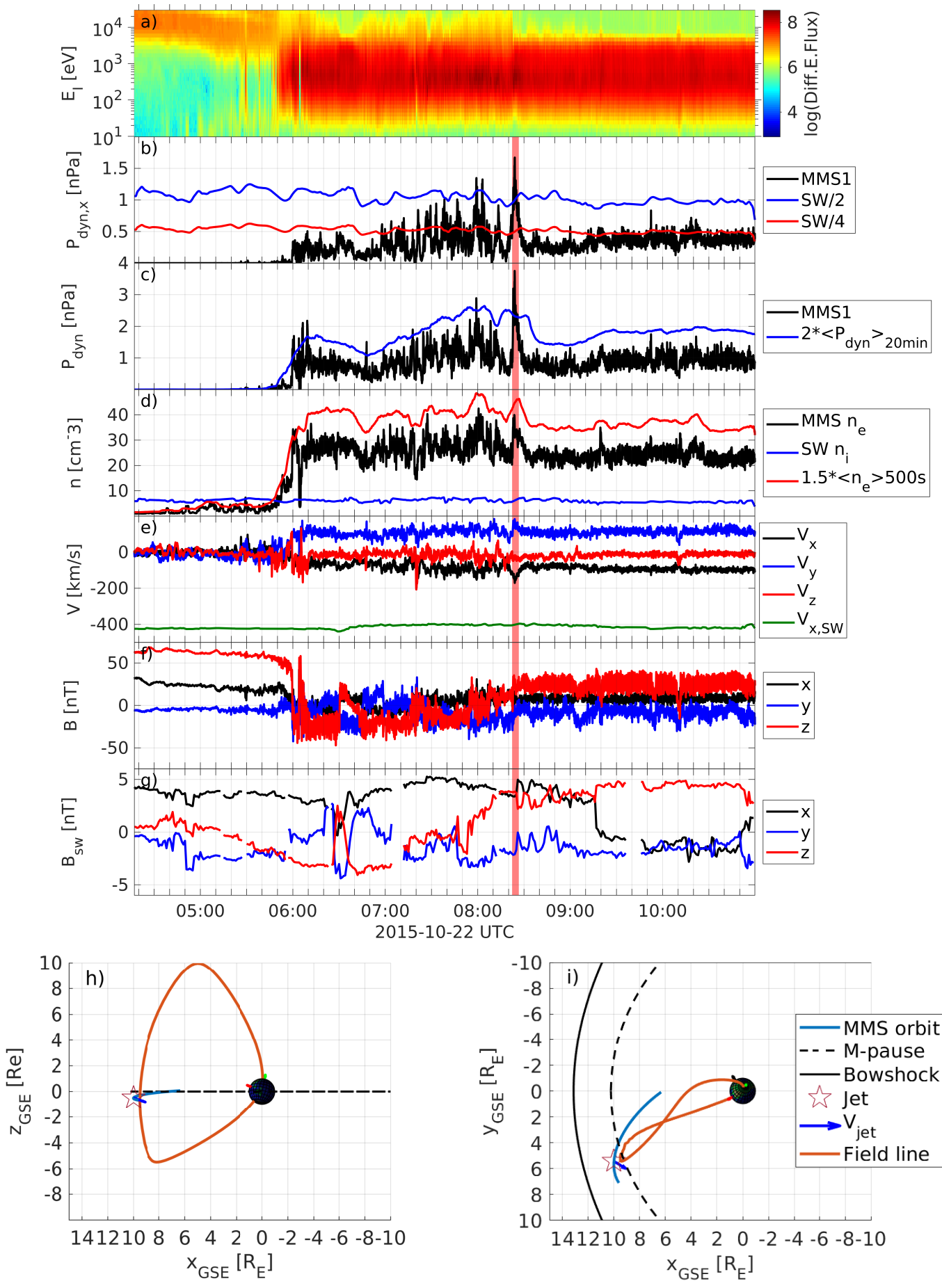

Figure 10: Panel a) Ion energy-time spectrogram from MMS 1, b) dynamic pressure in the $x_{G S E}$-direction from MMS 1 (black) and from OMNI (red and blue), c) omnidirectional dynamic pressure from MMS (black) and 20 min average (blue), d) electron density from MMS 1 (black) and $500 \mathrm{~s}$ average (red), ion density from OMNI (blue), e) ion velocity from MMS and OMNI, f) MMS magnetic field, g) IMF, h,i) $x y_{G S E}$ and $x z_{G S E}$ projection of MMS orbit and jet impact with the magnetopause. All OMNI data is propagated to the nominal bow shock. 


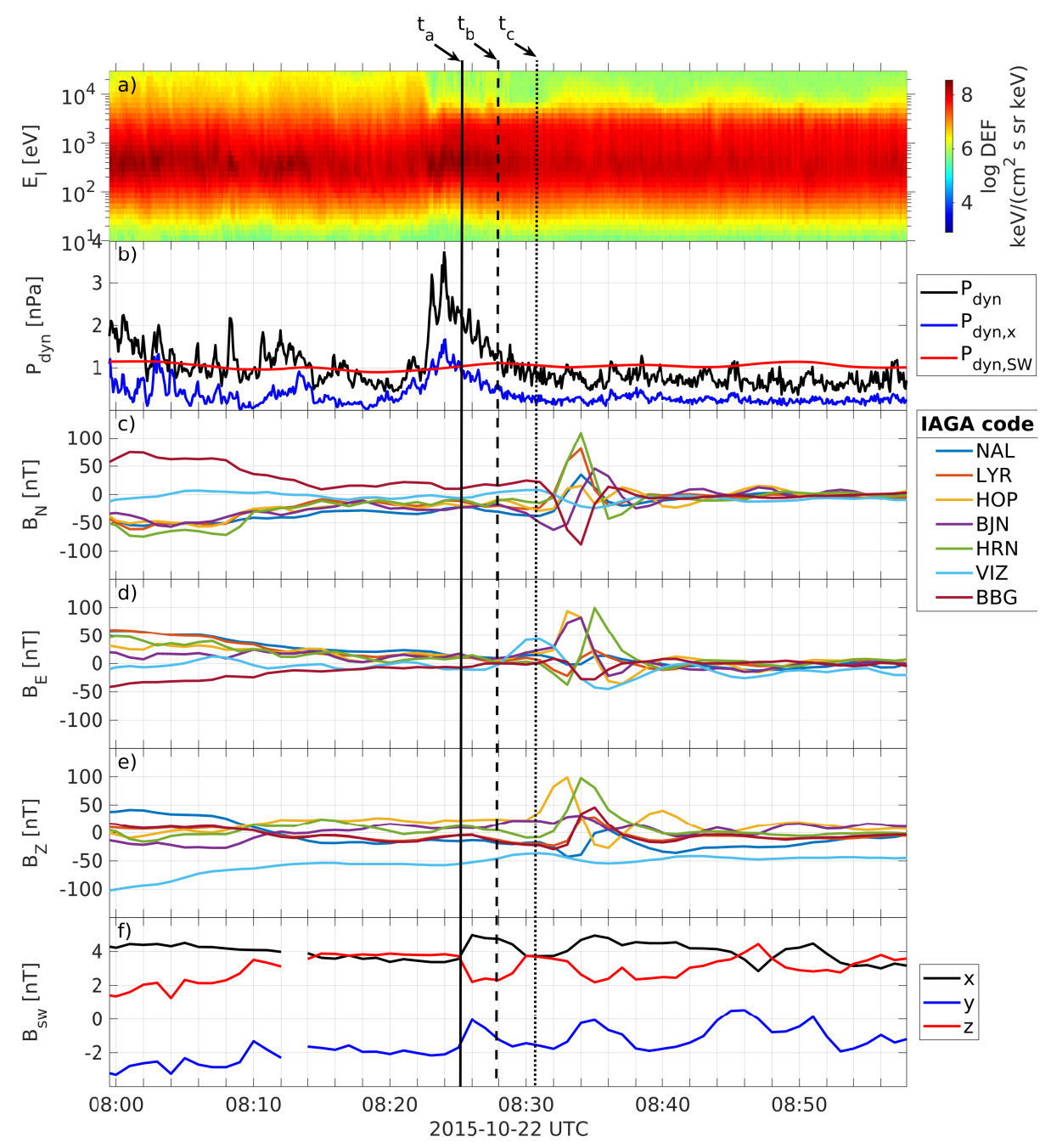

Figure 11: a) Ion energy time spectrogram from MMS, b) total dynamic pressure from MMS (black), only due to $v_{x, G S E}$ (blue) and upstream from OMNI (red), c)-e) $N, E$ and $Z$ components recorded by GMAGs (see Tab. 1), f) upstream magnetic field observations from OMNI. Time at the center of the jet, $t_{a}$, is marked with black solid line, first GMAG response, $t_{b}$, dashed line and later GMAG response as a dotted black line $t_{c}$. All OMNI data is propagated to the nominal bow shock.

Figure 11a-b shows a zoom of the ion energy-time spectrogram and dynamic pressure around the times of the detected jet. In the ion energy-time spectrogram, we see 
that the energies are more concentrated around $800-1000 \mathrm{keV}$ at the time of the jet, which is characteristic for these jets (see Sec. 5.1). The center of the jet, $t_{a}$, is marked at with a black solid line. Around $\sim 70 \mathrm{~s}$ after $t_{a}$, the GMAG station VIZ observed the beginning of a magnetic pulse in its $E$-component (Fig. 11d), marked with a black dashed line in Figure 11.

Around $70 \mathrm{~s}$ later, four other GMAG stations observed a similar magnetic pulse in all components (black dotted line in Fig. 11). All GMAGs observed an increase in the $N$-component except BBG, which instead observed a decrease in this component. We speculate that this could be an indication of a rotation of the magnetic field as observed at ground level during this magnetic pulse. The magnitude of this pulse is around $50 \mathrm{nT}$.

During the next selected event, MMS was inside the magnetosheath on an inbound orbit towards the Earth (see Fig. 12h-i). Looking at the ion energy-time spectrogram (Fig. 12a) we see similar behavior as in the previous case (Fig. 10), where MMS seems to be behind a quasi-parallel bow shock around 08:00-08:20 UTC, again indicated by the amount of high energy particle flux. After 08:20 UTC, this trend disappears and MMS observes indications of being behind a more quasi-perpendicular bow shock instead. At least until 08:30 UTC, there is a short period around 08:30-08:40 UTC the bow shock might be quasi-parallel again. Comparing this with the propagated OMNI observations (Fig. 10g), the IMF varies between 08:00-08:20 UTC. While afterwards it settles into a more stable northward configuration, with the exception of between 08:30-08:40. This is in good agreement with what MMS observes at the time.

The dynamic pressure as reported by MMS (Fig. 12b-c) was fluctuating during the same times as the IMF was varying, 08:00-08:20 UTC. Later, the fluctuations in the dynamic pressure was much smaller, and the magnetosheath seems to settle down as the bow shock changes from quasi-parallel to quasi-perpendicular. A similar behavior is also seen in the electron density, ion velocity and magnetic field (Fig. 12d-f) as observed by MMS.

Combining all three criteria (Fig. 12b-d, highlighted in red), MMS detected 3 jets between 07:50-08:30 UTC. Only Jet 2 was considered a jet according to the Plaschke criteria (Fig. 12b). Jet 1 and jet 3 showed sudden increase in $v_{y}$, while $v_{x}$ was the most increasing component in jet 2. Due to the position of MMS (Fig. 12h-i), all three jets was on collisional courses to the nominal magnetopause from T96. During all three jets, MMS observed changes in the magnetic field (Fig. 12f). However, during Jet 3 MMS observed a much larger magnetic field change than during the previous 2 jets. Jet 3 is also the most long lived one with a time duration of almost 3 minutes. 

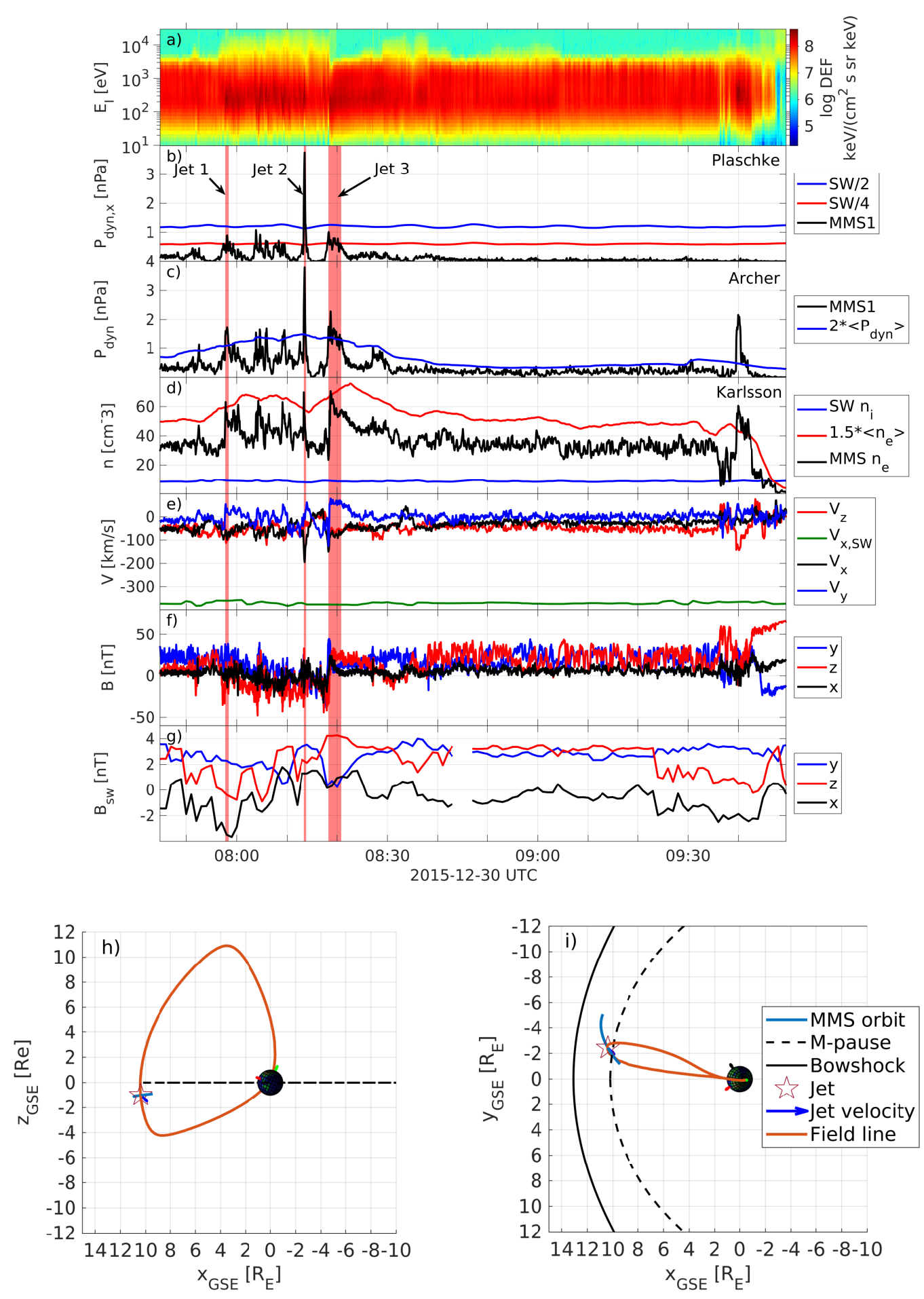

Figure 12: Similar description as 10 but for 2015-12-30. Three different jets are highlighted and marked labeled. 
Figure 13 shows a zoom around Jet 2 and Jet 3 (marked with arrows in panel b). Similar to the previous case (Fig. 11), it depicts the ion energy-time spectrogram, total dynamic pressure from MMS (black) and only due to $v_{x}$ (blue) and from the propagated OMNI observations (red). During both jets, we see similar trends as before in the spectrogram, where the energy flux is more concentrated around $800-1000 \mathrm{keV}$. The center of Jet 3 is marked with a black solid line. According to the theoretical estimations, the total transfer time needed for Jet 3 to cause perturbations in the ionosphere is around $50 \mathrm{~s}$ and the ionospheric foot-point is at $81^{\circ}$ latitude and $74.3^{\circ}$ longitude geographical coordinates according to the T96 model. Within a radius of $1800 \mathrm{~km}$ from this foot-point, 7 GMAG stations was operational at the time, the closest one being VIZ.

Some 2 min and $20 \mathrm{~s}$ after the center of Jet 2 ( $t_{a}$ in Fig. 13), GMAG station BJN observed the beginning of a magnetic pulse in all three components ( $t_{b}$ in Fig. 13). The $N$-component was slightly earlier compared to the $E$ - and $Z$-components. Around 2 min later ( $t_{c}$ in Fig. 13), the rest of the GMAGs, with the exception of VIZ, observed a similar magnetic pulse. VIZ does not show the same clear observations as the rest of the GMAGs at the time. It does however show a slight increase in the $N$-component at the same time as the center of the jet ( $t_{a}$ in Fig. 13), although with much lower amplitude.

Comparing the observed time $t_{a}$ to $t_{b}$ with the estimated transfer time (about $50 \mathrm{~s}$ ), the observed magnetic pulse in the GMAG observations is likely caused by Jet 3. Even though Jet 2 is stronger in dynamic pressure, its much shorter time duration is probably the cause for not being detected by the GMAGs. This is in agreement with what Archer et al. [3] suggest. 


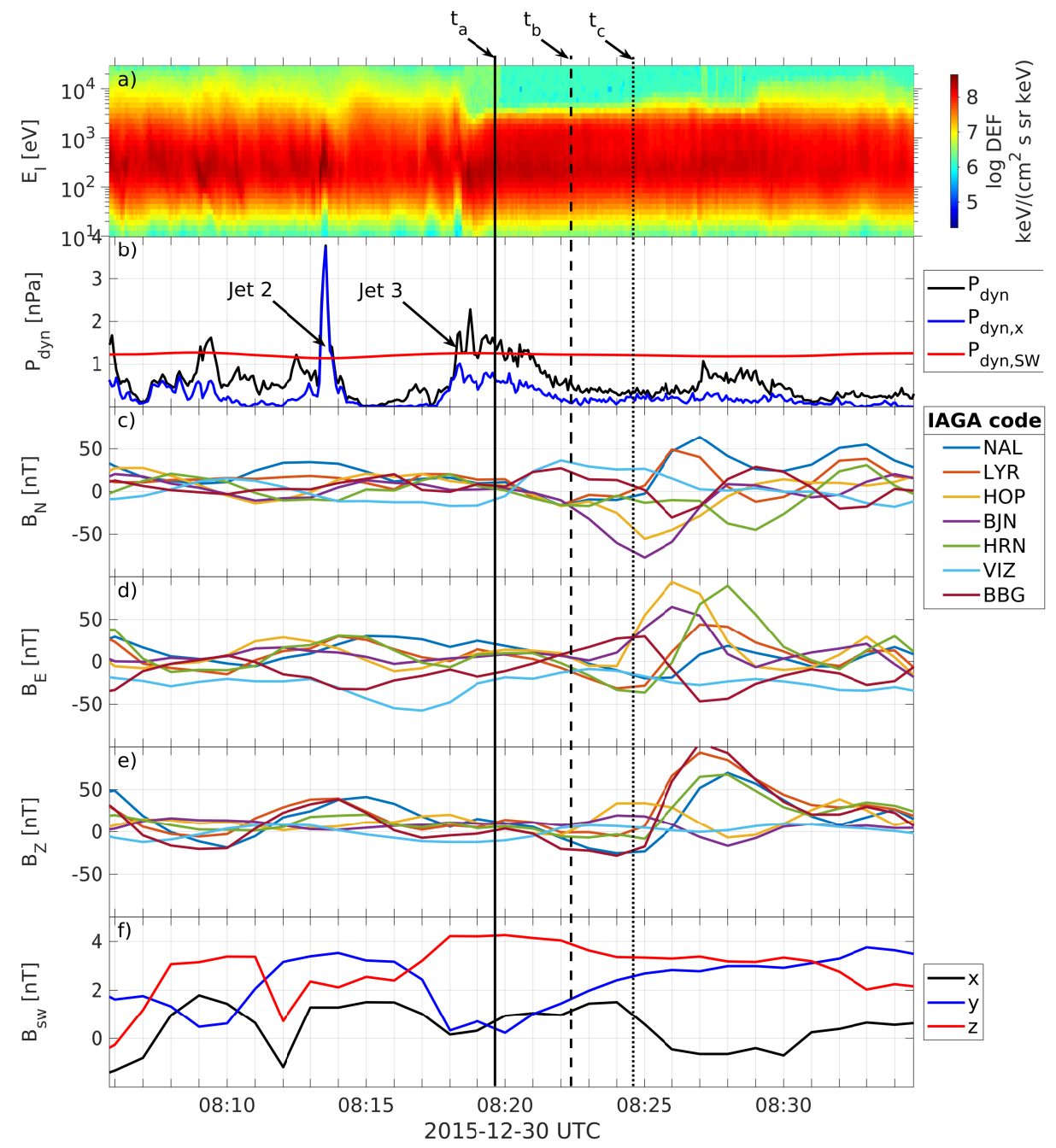

Figure 13: Similar description as 11 but for 2015-12-30. 
For our last presented case, which is from 2015-12-27 04:00-10:00 UTC, MMS was again in an inbound orbit towards Earth. MMS was inside the magnetosheath for almost the entire time duration, but did eventually reach the magnetosphere at the end (see Fig. 14). The magnetopause crossings at the end is visible Figure 14a, as brighter stripes in the spectrogram, indicating that MMS was near the magnetopause which moved relative to the spacecraft.

The ion energy-time spectrogram indicates that the bow shock changes from quasiparallel to quasi-perpendicular many times over the observed time interval, these periods are marked gray in Figure 14. Again, this is seen in the amount of observed high energy particles. The propagated OMNI data show multiple periods of stable radial IMF, due to MMS position (Fig. 14h-i), this is expected to be behind a quasi-parallel bow shock at. The MMS spectrogram and OMNI observations do not completely agree on whenever the bow shock is quasi-parallel. Due to the issues involved in propagating the OMNI data (see Sec. 4.2), the observations from MMS is likely more accurate.

All three used selection criteria detected multiple jets during the entire selected time interval visible in Figure 14, where most of them are observed behind a quasi-parallel bow shock or in the transitions between parallel to perpendicular (Fig. 14b-d). There is some jets observed in the quasi-perpendicular times as well, but they are much lower in strength. In general, all MMS observations show a lot more fluctuations during the quasi-parallel times, both in the dynamic pressure (Fig. 14b-d), electron density (Fig. 14e), velocity (Fig. 14f) and magnetic field readings (Fig. 14g).

The strongest of the observed jets, which appear slightly before 06:00 UTC, qualified as a jet according to all three criteria. In this jet, the plasma velocity $v_{x}$ reached around $-400 \mathrm{~km} / \mathrm{s}$, at the same time as the electron density jumped from $20 \mathrm{~cm}^{-3}$ to around $35 \mathrm{~cm}^{-3}$. This jet is marked in Figure $14 \mathrm{~h}-\mathrm{i}$ as a star, with its velocity as a blue arrow. 

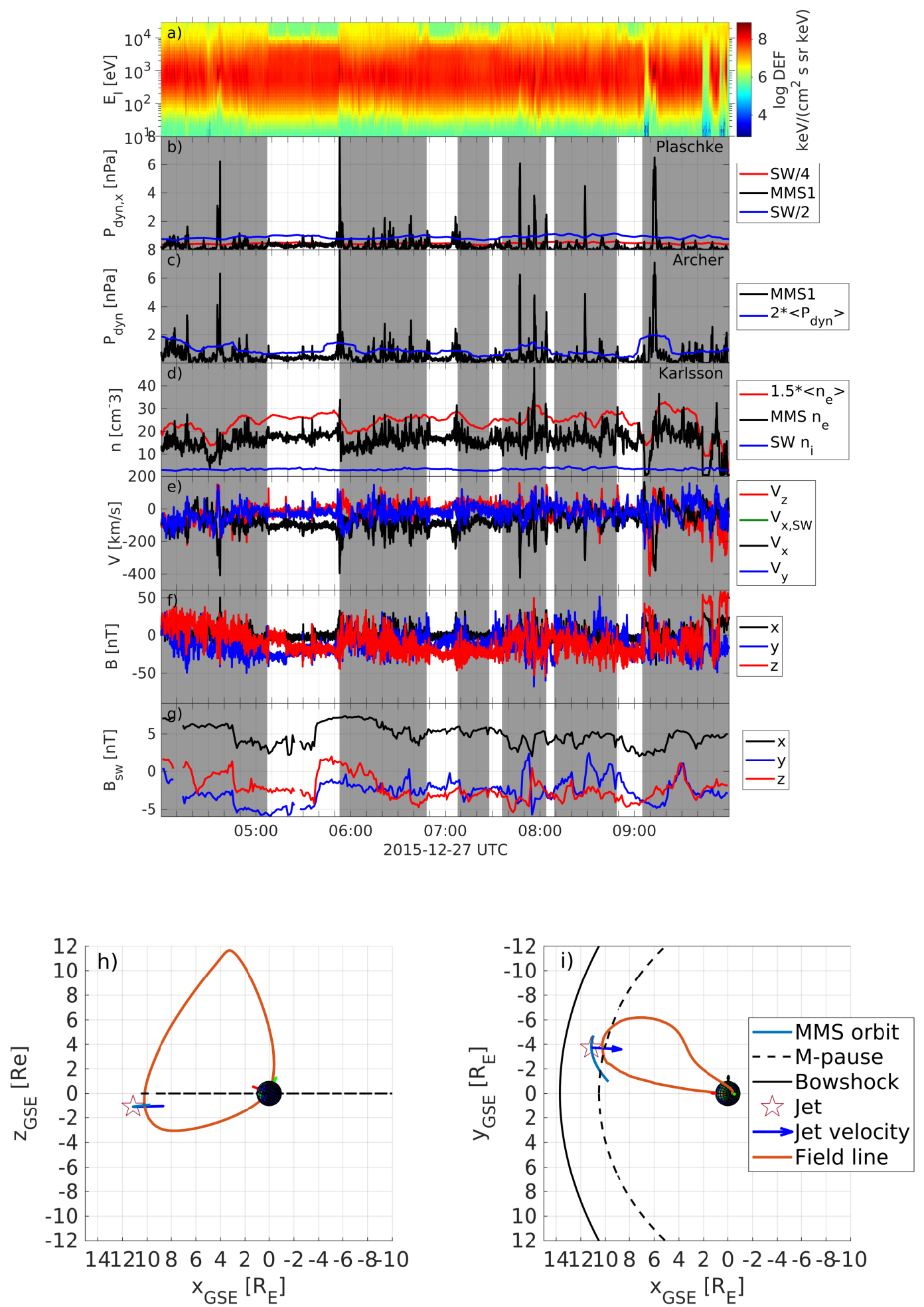

Figure 14: Description similar as 10 but for 2015-12-27. Periods where MMS lies behind a quasi-parallel bow shock is marked in gray. 


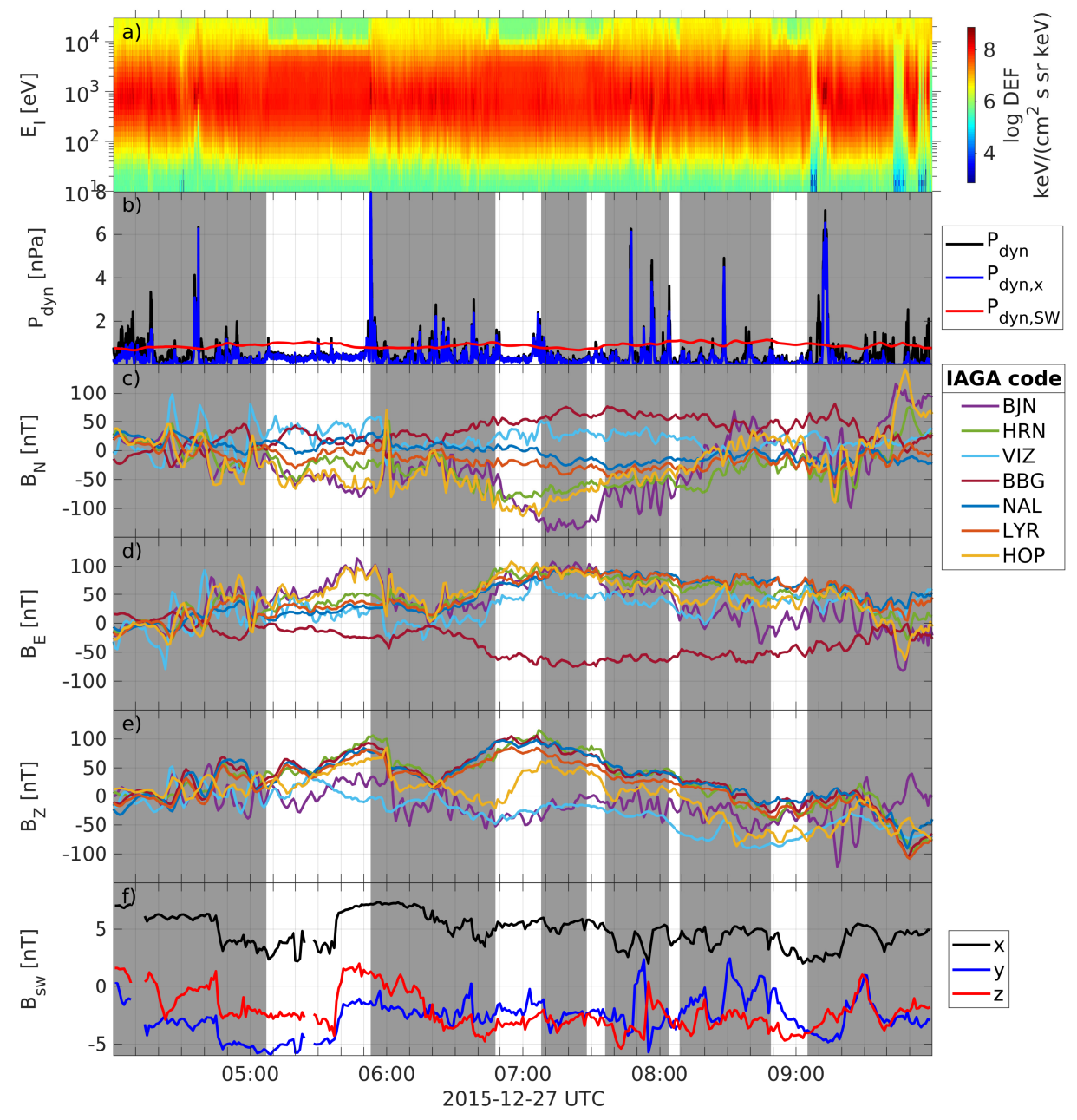

Figure 15: Similar figure as 11 but for 2015-12-27. Periods where MMS lies behind a quasi-parallel bow shock is marked in gray.

The jet at 06:00 UTC was propagated to the nominal bow shock from T96 and the affected field line had a ionospheric foot-point at $80.5^{\circ}$ latitude and $72.6^{\circ}$ longitude geographic coordinates. Within a radius of $1800 \mathrm{~km}, 7$ GMAG stations was operational at the time. Figure 15 shows the same time interval as Figure 14. Similarly as previous cases, panels $\mathrm{a}$ and $\mathrm{b}$ show the ion energy-time spectrogram and dynamic pressure respectively. The gray periods is the same as in Figure 14, i.e. when the spectrogram suggests that MMS was behind a quasi parallel bow shock. 
Figure 15c-d show the observations from the GMAG stations close to the foot-point. From these observations, it is seen that the GMAGs show a lot more fluctuations during the marked periods. The amplitude of the specific components is not necessarily larger, but they do show more variations whenever MMS was expected to lie behind a quasi-parallel bow shock. Since MMS was close to the nose of the bow shock during the entire time interval in Figure 15, this corresponds to a radial IMF configuration.

\subsection{Summary and discussion: case study}

In this section we have presented three cases with MMS, OMNI and GMAG observations. All presented cases suggest that some magnetosheath jets can be geoeffective. Most of our estimated foot-points have been found within a sector above $70^{\circ}$ magnetic latitude (see Sec. 3.1) and within \pm 2 hours of magnetic local noon ${ }^{9}$.

The first case from 2015-10-22, was an example of a jet detected in the magnetosheath. This jet was estimated to impact the magnetopause according to the T96 model. The affected field line had a foot-point in the arctic ocean, east of Svalbard, and the GMAG station VIZ observed a magnetic pulse about 1 min 20 s later. This transfer time is in good agreement with the theoretical estimated time for the jet to propagate the magnetopause, and for an Alfvén wave to travel along the field line down to the ionosphere. Additionally, four more nearby stations observed a similar magnetic pulse after additionally $1 \mathrm{~min} 20 \mathrm{~s}$, supporting that the observed pulse originates somewhere around our estimated foot-point. However, the T96 model might not be entirely accurate in tracing the field line, and thus also in predicting the coordinates of the foot-point, but the fact that five neighboring GMAGs observed similar signals indicates that the mapping was reasonable.

Our second presented case contained two separate jets, where one of them was stronger in dynamic pressure and had a shorter time duration. Even though both of the jets were predicted to impact the nominal magnetopause from T96, and likely also the actual magnetopause, only the more long-lived one seems to be related to the GMAGs responses. As previously mentioned, Archer et al. [3] (2013) suggested that the magnetopause can act as a low pass-filter. According to that, only perturbations of long enough time durations should be detectable inside the magnetosphere and ionosphere. This might help explain why the more long-lived jet was the one most likely to be detected by the GMAGs.

\footnotetext{
${ }^{9}$ Magnetic local noon approximately corresponds to the places on Earth with local noon, i.e. the sun appears to lie along the $x_{G S E}$-axis.
} 
Lastly, we presented a case over a longer time interval of steady radial and non-radial IMF. This case contained multiple jets, especially during the radial IMF periods. We selected one of the jets and estimated at which foot-point it could be detected by GMAGs. This GMAG and other stations close to this foot-point observed more fluctuations during the periods of radial IMF compared to the non-radial periods. During radial IMF, the nose of the bow shock will be in a quasi-parallel state. It is suggested that jets are more commonly created behind such a bow shock. This is supported by our presented case. We suggest that more jets are likely to impact the magnetopause during this IMF configuration, and that it is this increased amount of impacting jets that are causing the GMAGs to observe variations in the ionosphere.

Our cases suggests that the observed total transfer time is around 1-2 min, from jet detection in the magnetosheath to the GMAGs observing the beginning of a magnetic pulse. We are however limited by the temporal resolution of the GMAG data, which is only 1 data point per minute. This makes it difficult to estimate where the GMAG response begins. Furthermore, signals which are shorter than 1 min will be difficult to detect among GMAG data. For example, in our second case (Fig. 13), Jet 2 might be causing disturbances which could be detected at ground, if it is not low-pass filtered out by the magnetosphere [3].

Worth noting, is that among all these investigated cases, not all jets resulted in a clearly resolved responses in GMAGs. This can be explained by the lack of GMAGs at the actual foot-points for certain cases, the low temporal resolution provided by SuperMag. It could of course also be the case that they simply do not impact the magnetopause, or possibly, impact the magnetopause but do not show any geoeffectiveness. For further studies, higher time resolution among the GMAGs should be considered. This can be done for certain groups of GMAGs but not all of them. We also aim to investigate the cases not presented here in further depth for future studies, as well as locating more cases among spacecraft observations.

\section{Statistical study of IMF orientation}

As seen in our last presented case (Fig. 15), observations from GMAGs seem to fluctuate more during radial IMF. During this IMF orientation, jets are suggested to be more common [23]. To investigate if this is a general behavior, that is, GMAG observations show more fluctuations during radial IMF, we performed a statistical investigation comparing IMF orientation with GMAG observations.

In this section we will present this statistical investigation. First we will describe our method, how different IMF orientations were chosen and which GMAGs were 
selected for comparison. Then we present our statistical results followed by a discussion and summary.

\subsection{Method}

We used data from the THEMIS spacecraft for surveying the IMF instead of the otherwise commonly used OMNI data, see Sections 4.2 and 4.3. Data from THEMIS was selected whenever the spacecraft were more than $17 R_{E}$ in the $x_{G S E}$ direction, to make sure that we obtained observations upstream the bow shock. These data were propagated to the nominal bow shock, see Section 4.3.

Radial IMF is defined as whenever the angle between the IMF field lines and the $x_{G S E}$-axis is less than $30^{\circ}$. We also distinguished between radially Earthward or sunward by the sign of $B_{x}$ being negative or positive respectively. Northward and southward IMF was defined as whenever $B_{z}$ was positive or negative respectively, and the dominating (largest magnitude) component. To ensure that we used stable IMF observations, time periods of at least 30, 40, 50, 60, 70, 80, and 90 min long intervals with steady IMF was selected. This was done by requiring that, once an IMF data point from THEMIS was found to belong to one of the orientations, data points from all the preceding 30-90 min should also belong to the same orientation. The left part of Figure 16 shows a period (marked green/red) of radially Earthward $\left(B_{x}<0\right)$ IMF located by THEMIS B.

Since changes in the IMF orientation is sometimes related to discontinuities and very sudden changes which might affect the magnetosphere, we wanted to exclude such effects observed by the GMAGs. For every selected time interval, we therefore removed the first and last $10 \mathrm{~min}$ from the period, displayed as red markings in the left panel of Figure 16.

From our case study in the previous chapter, we found that most foot-points were located above $70^{\circ}$ magnetic latitude and within \pm 2 hours of magnetic local noon. In this statistical study, we therefore selected GMAGs within this region on the northern hemisphere, see the red marked circle segment in the right panel of Figure 16.

The observations from the selected GMAGs were sorted to belong to a northward, southward, radially Earthward or radially sunward IMF orientation. To estimate the amount of variation in the GMAG observations, the standard deviation $\sigma$ was calculated, for all the available stations, both for their individual components, $N, E$, and $Z$ (see Sec. 3.1) as well as for the magnitude. We did this for all IMF orientations and all 30-90 min time length intervals. Finally, the mean values of the observed standard deviations were calculated. We complemented these mean values with their 
standard errors, $\sigma_{x}=\sigma / \sqrt{n}, n$ being the number of data points, to get an estimate of the accuracy in them. This way we had indications of the average variations during different IMF orientations with some statistical certainty. The variation of the magnitude is especially interesting since the magnitude is proportional to the energy density in a magnetic field.
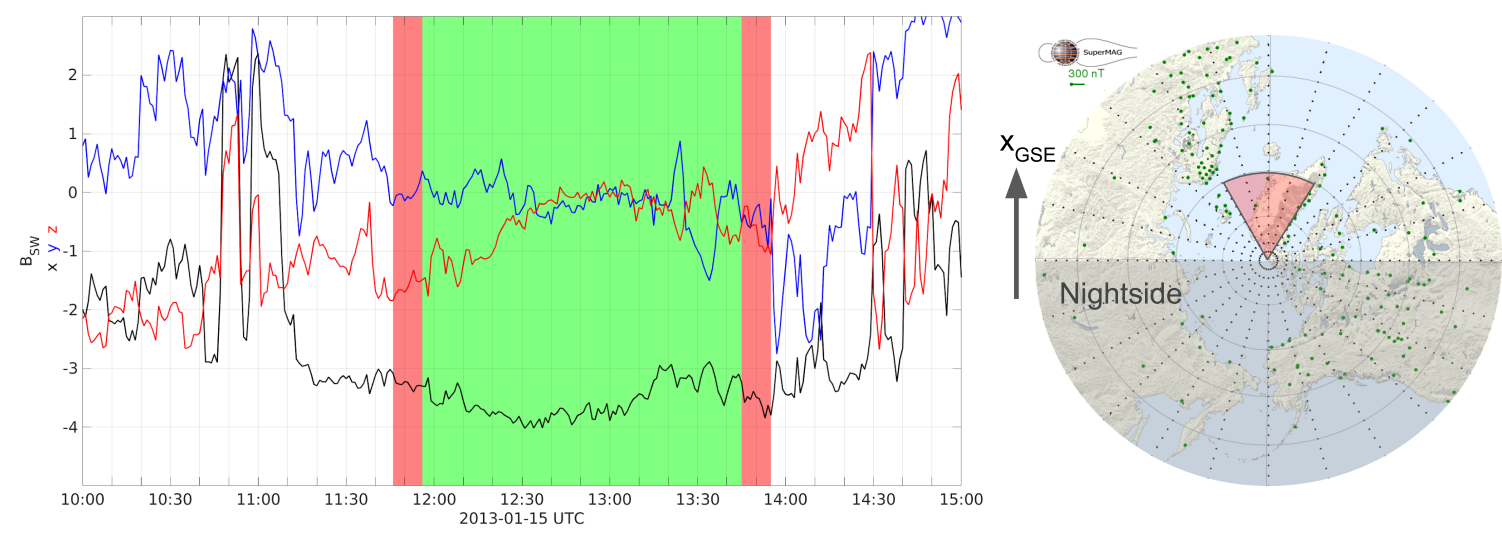

Figure 16: Left: IMF observations by THEMIS B 2013-01-15, a period of radially Earthward IMF is highlighted in green/red, where the red parts are the first/last 10 minutes of the period. Right: polar map showing GMAGs as green dots and the region from where we selected GMAGs in red, adopted from [26].

\subsection{Observations}

Figure 17 displays the result of our statistical study. The panels show the calculated standard deviations as colored bars, from left to right, of $B_{N}, B_{E}, B_{Z}$, and $|\mathbf{B}|$, as observed by GMAGs selected according to our above mentioned criteria (red circle segment to the right in Fig. 16). Error bars corresponding to $\pm 2 \sigma_{x}$ of the standard deviations are also shown. The $x$-axis on each panel correspond to the different minimum time durations used for selecting IMF intervals, while the different colors represent GMAG observations during the four different IMF orientations, radially sunward (yellow), radially Earthward (green), norhtward (red), and southward (blue).

During radial IMF, both sun- and Earthward (yellow and green bars in Fig. 17), the GMAG observations appear to fluctuate more than during northward IMF (red bars). This holds for all components as well as for the magnitude. For times of at least $50 \mathrm{~min}$, the observed fluctuations from GMAGs is slightly higher for radially 
sunward (yellow bars) IMF compared to radially Earthward (green bars), at least for the $N$-components and the magnitude. We were expecting that the fluctuations of the GMAG observations would be higher at all times during sunward IMF compared to Earthward. Comparing with Figure 3c-d, we see that during radially sunward IMF we get a reconnection site, on the northern hemisphere, at a similar region as during southward IMF. According to the results, this is not allays the case (yellow bars sometimes smaller than the green bars) and we are not sure of why this is.

The observed fluctuations in GMAGs is considerably higher during southward IMF (blue bars), as is expected due to dayside magnetopause reconnection (see Fig. 3 Sec. 2.1). The fluctuations also increase as the time intervals get longer. This suggests that the disturbances within the magnetosphere gets higher the longer IMF is in a southward orientation, which is as expected. Note that, since our selected time intervals of 30-90 min are minimum durations of steady IMF, everything in the 40-90 min intervals are included in the $30 \mathrm{~min}$ intervals as well (and so on). Hence, the data sets are larger for the shorter time intervals, resulting in smaller error-bars compared to the longer intervals.
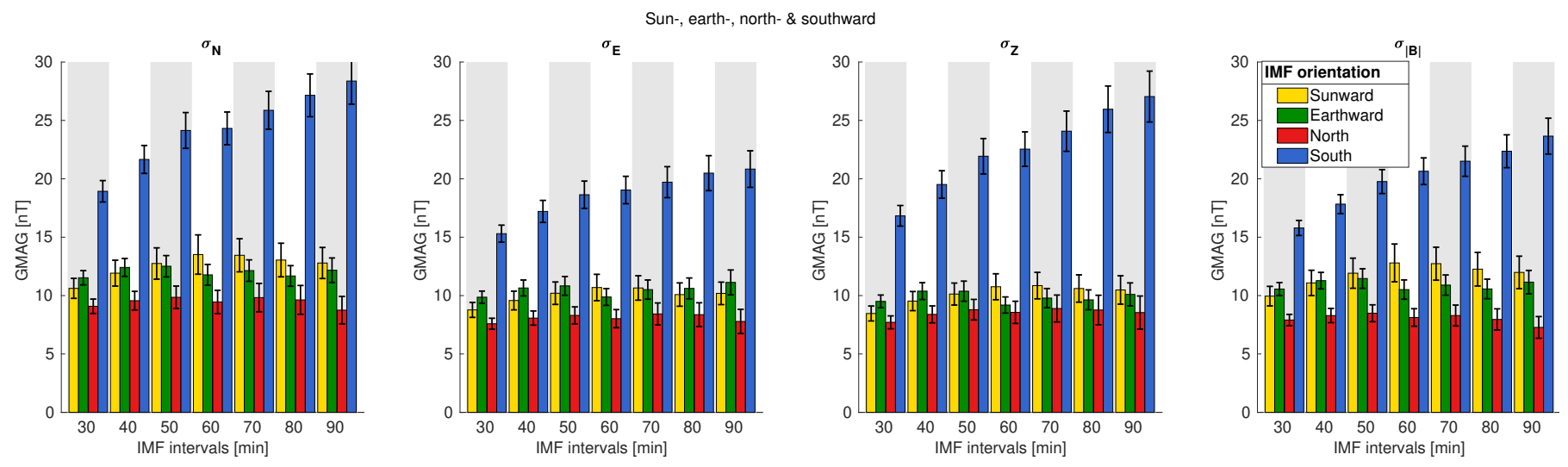

Figure 17: Standard deviations of GMAG observations (with error bars) during sun-, Earth-, north- and southward IMF for our 30-90 min time length intervals. The three panels to the left depicts the standard deviation of the $N, E$, and $Z$-components respectively, as observed by GMAGs, while the rightmost panel show the standard deviation of $|\mathbf{B}|$.

Also worth noting, is that the standard deviation of the magnitude during southward IMF (rightmost panel Fig. 17) is generally smaller than the standard deviation for some of the components. This might be confusing at first, since the magnitude is just the sum of the squares of each component, if the components vary the magnitude should vary equally. But one can imagine a case where the observed magnetic 
vector is just rotating around, causing the components to vary, while the magnitude remains more stable. This would give a result where the standard deviation of the components is higher than that of the magnitude.

\subsection{Summary and discussion: statistical study}

In this section we have presented a statistical investigation regarding variations in GMAG observations at the dayside, as determined by their standard deviations. We compared the amount of fluctuations during periods of stable IMF with radially sunward, radially Earthward, northward, and southward orientations.

The results indicate that during radial IMF, both sun- and Earthward, dayside GMAGs above a certain latitude observe more fluctuations compared to northward IMF. This is in line with what Figure 15 suggests: GMAGs close to the foot-point observed higher fluctuations at times when MMS was behind a quasi-parallel bow shock (corresponding to radial IMF in that case). As previously mentioned, jets close to the bow shock nose is more common during radial IMF. The additional fluctuations observed by GMAGs during radial IMF, as shown in Figure 17, could be explained by the increased amount of jets impacting the magnetopause and disturbing the magnetosphere.

The IMF observations were provided by THEMIS B whenever it was upstream of the Earth's bow shock. These observations were time-shifted to the nominal bow shock in a very simple manner. For further studies, this method of propagation should be refined to take into account THEMIS actual position, as compared to the average position. Using the T96 model to locate the averaged positions of foot-points could also refine our selection of GMAGs in a statistical way.

\section{Summary and conclusions}

In this project we have examined the geoeffectiveness of magnetosheath jets. This has been done by comparing GMAG observations with in-situ detection of jets, and by a statistical investigation comparing IMF orientation with variations in GMAG observations. From the cases presented in section 5.3, it seems likely that jets can cause perturbations detectable at ground level by GMAGs. Previous studies also report cases with effects caused by jets on ground based observations, for example $[3,9,14]$. However, only some of the previous studies have used GMAGs for ground based comparisons. In some studies, radar and optical observations have been used as instead. 
We suggest that jets can be geoeffective and cause ground based disturbances detectable by GMAGs. This is supported by our case study where we found that the theoretical transfer times, from jet detection to expected GMAG responses, were in good agreement with the observed times which was around 1-2 min. Furthermore, the results from our statistical study also supports that jets can cause geoeffective disturbances detectable by GMAGs.

From our case study, we find indications that jets of short time lengths do not give any response in GMAG observations. This is in agreement with the magnetopause acting as a low pass filter, as suggest by Archer et al. [3] (2013). We suggest further studies to examine this more in depth, by locating more cases and comparing with additional GMAG observations, preferably with higher time resolution. We would also like to expand on our statistical study, by utilizing similar models as T96, and trace field lines to get a statistical sense of where foot-points to field lines at the subsolar point ends up. This would allow a more refined way of selecting GMAG stations.

In conclusion, jets can be geoeffective. Understanding geoeffective phenomena upstream Earth's bow shock is important for a better understanding of space weather. Jets show $\mathrm{d} \mathbf{B} / \mathrm{d} t$-effects at ground level, which can induce currents in conducting materials close to the foot-points. In our found cases however, the values of these effects ar very small, in the order of $1 \mathrm{nT} / \mathrm{s}$, which is not that impactful. But higher resolution in GMAG data might well increase this value, and it is therefore of interest to investigate this further.

\section{Acknowledgments}

We acknowledge the use of NASA/GSFC's Space Physics Data Facility's OMNIWeb (or CDAWeb or ftp) service, and OMNI data. We would also like to thank SuperMAG for the use of their provided data from SuperMAGweb [26]. We acknowledge the use of GMAG data from the collaborators in the IMAGE chain for the ground based observations around Svalbard. 


\section{References}

[1] H. Alfvén. Existence of Electromagnetic-Hydrodynamic Waves. Nature, 150(3805):405-406, Oct 1942.

[2] M. O. Archer and T. S. Horbury. Magnetosheath dynamic pressure enhancements: occurrence and typical properties. Annales Geophysicae, 31(2):319-331, 2013.

[3] M. O. Archer, T. S. Horbury, J. P. Eastwood, J. M. Weygand, and T. K. Yeoman. Magnetospheric response to magnetosheath pressure pulses: A lowpass filter effect. Journal of Geophysical Research: Space Physics, 118(9):54545466, 2013.

[4] W. Baumjohann and R. A. Treumann. Basic Space Plasma Physics. Imperial College Press, 1997.

[5] M. Bester, M. Lewis, B. Roberts, and D. Cosgrove. Operations planning and mission readiness testing for the themis spacecraft constellation. IEEE Aerospace Conference Proceedings, 032010.

[6] J. L. Burch, T. E. Moore, R. B. Torbert, and B. L. Giles. Magnetospheric multiscale overview and science objectives. Space Science Reviews, 199(1):5-21, Mar 2016.

[7] M. Chiu, U. Von-Mehlem, C. Willey, T. Betenbaugh, J. Maynard, J. Krein, R. Conde, W. Gray, J. Hunt, L. Mosher, M. McCullough, P. Panneton, J. Staiger, and E. Rodberg. Ace spacecraft. Space Science Reviews, 86(1):257284, Jul 1998.

[8] A. Dmitriev and A. Suvorova. Large-scale jets in the magnetosheath and plasma penetration across the magnetopause: Themis observations. Journal of Geophysical Research: Space Physics, 052015.

[9] A. V. Dmitriev and A. V. Suvorova. Traveling magnetopause distortion related to a large-scale magnetosheath plasma jet: Themis and ground-based observations. Journal of Geophysical Research: Space Physics, 117(A8), 2012.

[10] J. W. Gjerloev. A Global Ground-Based Magnetometer Initiative. Eos, 90:230231, jul 2009.

[11] J. W. Gjerloev. The supermag data processing technique. Journal of Geophysical Research: Space Physics, 117(A9), 2012. 
[12] H. Gunell, H. Nilsson, G. Stenberg, M. Hamrin, T. Karlsson, R. Maggiolo, M. Andr, R. Lundin, and I. Dandouras. Plasma penetration of the dayside magnetopause. Physics of Plasmas, 19(7):072906, 2012.

[13] H. Gunell, G. Stenberg Wieser, M. Mella, R. Maggiolo, H. Nilsson, F. Darrouzet, M. Hamrin, T. Karlsson, N. Brenning, J. De Keyser, M. André, and I. Dandouras. Waves in high-speed plasmoids in the magnetosheath and at the magnetopause. Annales Geophysicae, 32(8):991-1009, 2014.

[14] D.-S. Han, H. Hietala, X.-C. Chen, Y. Nishimura, L. R. Lyons, J.-J. Liu, H.-Q. $\mathrm{Hu}$, and H.-G. Yang. Observational properties of dayside throat aurora and implications on the possible generation mechanisms. Journal of Geophysical Research: Space Physics, 122(2):1853-1870, 2017.

[15] H. Hietala, N. Partamies, T. Laitinen, L. Clausen, G. Facsk, A. Vaivads, H. Koskinen, I. Dandouras, H. Reme, and E. Lucek. Supermagnetosonic subsolar magnetosheath jets and their effects: From the solar wind to the ionospheric convection. Annales Geophysicae, 30:33-48, 012012.

[16] H. Karimabadi, V. Roytershteyn, H. X. Vu, Y. A. Omelchenko, J. Scudder, W. Daughton, A. Dimmock, K. Nykyri, M. Wan, D. Sibeck, M. Tatineni, A. Majumdar, B. Loring, and B. Geveci. The link between shocks, turbulence, and magnetic reconnection in collisionless plasmas. Physics of Plasmas, 21(6):062308, 2014.

[17] T. Karlsson, N. Brenning, H. Nilsson, J.-G. Trotignon, X. Vallires, and G. Facsko. Localized density enhancements in the magnetosheath: Three-dimensional morphology and possible importance for impulsive penetration. J. Geophys. Res., 117(A03227):891-921, 2012.

[18] T. Karlsson, A. Kullen, E. Liljeblad, N. Brenning, H. Nilsson, H. Gunell, and M. Hamrin. On the origin of magnetosheath plasmoids and their relation to magnetosheath jets. Journal of Geophysical Research: Space Physics, 120(9):73907403, 2015.

[19] NASA. Nasa/gsfc's omni data set through omniweb. https://spdf.gsfc. nasa.gov/pub/data/omni/, 2000.

[20] K. Ogilvie and M. Desch. The wind spacecraft and its early scientific results. Advances in Space Research, 20(4):559 - 568, 1997. Results of the IASTP Program.

[21] F. Plaschke, H. Hietala, and V. Angelopoulos. Anti-sunward high-speed jets in the subsolar magnetosheath. Ann. Geophys., 31(10):1877-1889, 2012. 
[22] F. Plaschke, H. Hietala, and V. Angelopoulos. Anti-sunward high-speed jets in the subsolar magnetosheath. Annales Geophysicae, 31(10):1877-1889, 2013.

[23] F. Plaschke, H. Hietala, M. Archer, X. Blanco-Cano, P. Kajdič, T. Karlsson, S. H. Lee, N. Omidi, M. Palmroth, V. Roytershteyn, D. Schmid, V. Sergeev, and D. Sibeck. Jets downstream of collisionless shocks. Space Science Reviews, 214(5):81, Jun 2018.

[24] C. Pollock, T. Moore, A. Jacques, J. Burch, U. Gliese, Y. Saito, T. Omoto, L. Avanov, and et al. Fast plasma investigation for magnetospheric multiscale. Space Science Reviews, 199(1):331-406, Mar 2016.

[25] J.-H. Shue, J. Chao, H. Fu, P. Song, K. Khurana, and H. Singer. A new functional form to study the solar wind control of the magnetopause size. Journal of Geophysical Research, 102:9497-9512, 011997.

[26] SuperMAG. http://supermag.jhuapl.edu/mag/?fidelity=low\&start= 2015-10-19T10\%3A12\%3A00.000Z\&interval=05\%3A00, 2000.

[27] R. B. Torbert, C. T. Russell, W. Magnes, R. E. Ergun, P.-A. Lindqvist, O. LeContel, H. Vaith, J. Macri, S. Myers, D. Rau, J. Needell, B. King, M. Granoff, M. Chutter, I. Dors, G. Olsson, Y. V. Khotyaintsev, A. Eriksson, C. A. Kletzing, S. Bounds, B. Anderson, W. Baumjohann, M. Steller, K. Bromund, G. Le, R. Nakamura, R. J. Strangeway, H. K. Leinweber, S. Tucker, J. Westfall, D. Fischer, F. Plaschke, J. Porter, and K. Lappalainen. The fields instrument suite on mms: Scientific objectives, measurements, and data products. Space Science Reviews, 199(1):105-135, Mar 2016.

[28] R. A. Treumann and W. Baumjohann. Advanced Space Plasma Physics. Imperial College press, 1997.

[29] N. A. Tsyganenko. Effects of the solar wind conditions in the global magnetospheric configurations as deduced from data-based field models (Invited). In E. J. Rolfe and B. Kaldeich, editors, International Conference on Substorms, volume 389 of ESA Special Publication, page 181, Oct 1996.

[30] A. A. Vlasov. The vibrational properties of an electron gas. Soviet Physics Uspekhi, 10(6):721-733, jun 1968. 